\title{
Vector bundles on genus 2 curves and trivectors
}

\author{
Eric M. Rains and Steven V Sam
}

\begin{abstract}
Given a complex curve $C$ of genus 2, there is a well-known relationship between the moduli space of rank 3 semistable bundles on $C$ and a cubic hypersurface known as the Coble cubic. Some of the aspects of this are known to be related to the geometric invariant theory of the third exterior power of a 9-dimensional complex vector space. We extend this relationship to arbitrary fields and study some of the connections to invariant theory, which will be studied more in-depth in a followup paper.
\end{abstract}

\section{Introduction}

Let $C$ be a smooth genus 2 curve over a field $k$ with a Weierstrass point $P$ defined over $k$. In the case when $k$ is algebraically closed and of characteristic 0 , the following situation has been studied in [Ngu07, Ort05]. First, using $(C, P)$, we get a canonically defined divisor $\Theta$ on the Jacobian $\mathrm{J}(C)$ of degree 0 line bundles on $C$. We correspondingly get an embedding $\mathrm{J}(C) \subset|3 \Theta|^{*} \cong \mathbb{P}^{8}$. Then there exists a unique cubic hypersurface in $\mathbb{P}^{8}$, the Coble cubic of $C$, whose singular locus is $\mathrm{J}(C)$ (see [Bar95, Cob17] and also [Bea03] for a concise proof). Furthermore, let $\mathrm{SU}_{3}(C)$ be the moduli space of semistable rank 3 bundles on $C$ with trivial determinant. Then there is a natural surjective map $\mathrm{SU}_{3}(C) \rightarrow|3 \Theta|$ of degree 2 (see Section 3) whose branch locus is a sextic hypersurface. Dolgachev conjectured that these two hypersurfaces are projectively dual to one another, and this was proven in [Ort05] and independently in [ $\mathrm{Ngu07}]$.

In [GS15, GSW13], it was shown that the construction of the Coble cubic is closely connected to the invariant theory of the group $\mathrm{SL}_{9}(k)$ acting on $\bigwedge^{3}\left(k^{9}\right)$ and also of the group $\mathrm{SL}_{8}(k)$ acting on $\bigwedge^{2}\left(k^{8}\right)$. In fact, the construction associates with each stable element in $\bigwedge^{3}\left(k^{9}\right)$ a smooth curve of genus 2 , and this construction is surjective on moduli. This naturally leads to the question of whether one can construct the trivector from the curve. This question is studied in [RS17], where the approach is relatively elementary, but requires one to solve an overdetermined system of linear equations to reconstruct the trivector, and it would thus be of interest to have a more direct construction.

The construction in [GS15, GSW13] is based on viewing an element of $\bigwedge^{3}\left(k^{9}\right)$ as a family of elements of $\Lambda^{2}\left(k^{8}\right)$ (that is, as a section of $\Omega^{2}(3)$ on $\left.\mathbb{P}^{8}\right)$, but we can equally well view it as a family of elements of $\Lambda^{3}\left(k^{8}\right)$ (that is, as a section of $\Lambda^{3} Q$, where $Q$ is the tautological quotient bundle on $\left.\mathbb{P}^{8}\right)$. In characteristic 0 , the stabilizer of a generic element of $\mathbb{P}\left(\bigwedge^{3}\left(k^{8}\right)\right)$ in

Received 23 February 2017, accepted in final form 22 January 2018.

2010 Mathematics Subject Classification 14H60, 15A72.

Keywords: genus 2 curves, vector bundles, invariant theory, moduli spaces.

This journal is (C) Foundation Compositio Mathematica 2019. This article is distributed with Open Access under the terms of the Creative Commons Attribution Non-Commercial License, which permits non-commercial reuse, distribution, and reproduction in any medium, provided that the original work is properly cited. For commercial re-use, please contact the Foundation Compositio Mathematica.

Steven V Sam was partially supported by a Miller research fellowship and NSF DMS-1500069. 


\section{VECTOR BUNDLES ON GENUS 2 CURVES AND TRIVECTORS}

$\operatorname{PGL}_{9}(k)$ can be shown to be (geometrically) isomorphic to Aut $\left(\mathfrak{s l}_{3}\right)$ (this was shown implicitly in $[\mathrm{LR} 79, \S 6.2]$, where the projective normalizer in $\mathrm{SL}_{8}$ was computed; for an alternate proof valid in arbitrary characteristic, see Proposition 2.7 and Corollary 2.8 below) and to be the unique trivector stabilized by that group. Since this group has two components, this induces a natural double cover of $\bigwedge^{3}\left(k^{8}\right)$, which in our setting pulls back to a double cover of $\mathbb{P}^{8}$, which furthermore comes with a (generic) identification of its tangent spaces with $\mathfrak{s l}_{3}$. This suggests both that this double cover of $\mathbb{P}^{8}$ should be a moduli space of rank 3 vector bundles (in particular, $\mathrm{SU}_{3}(C)$, since this is known to be a double cover of $\mathbb{P}^{8}$ ) and that we should be able to reconstruct the trivector from a suitable family of trivectors in the tangent bundle of $\mathrm{SU}_{3}(C)$.

Our aim in the present article is to make these heuristics precise and to extend these results to an arbitrary ground field $k$. Namely, we show that there is a degree 2 map $\mathrm{SU}_{3}(C) \rightarrow|3 \Theta|$ which is branched along an irreducible sextic hypersurface. Outside of characteristic 2, this is a reduced hypersurface, and its projective dual is a cubic hypersurface whose singular locus is isomorphic to $\mathrm{J}(C)$ (Theorem 5.4); for characteristic 2, see Remark 5.8. We also explain how all of these schemes may be constructed directly from a vector $\gamma \in \bigwedge^{3}\left(k^{9}\right)$ which is stable with respect to the action of $\mathrm{SL}_{9}(k)$.

\section{Invariant theory of $\wedge^{3}(8)$}

Let $V_{8}$ be an 8-dimensional vector space. Consider the action of $\operatorname{GL}\left(V_{8}\right)$ on $\bigwedge^{3} V_{8}$. There is a unique $\mathrm{GL}\left(V_{8}\right)$-invariant hypersurface if $k$ has characteristic 0 , its degree is 16 , and $\mathrm{GL}\left(V_{8}\right)$ acts transitively on its complement (when $k=\bar{k}$ ) [SK77, $\S 5$, Proposition 10]. So we can clear denominators to get a multiple that is defined over $\mathbb{Z}$ (we will isolate a particular multiple in Lemma 2.2). We call this hypersurface the hyperdiscriminantal locus.

For any $n$, there is a natural trilinear form $\bigwedge^{3} \mathfrak{p g l}_{n} \rightarrow k$ defined by

$$
\left(X_{1}, X_{2}, X_{3}\right) \mapsto \operatorname{Tr}\left(\widetilde{X}_{1} \widetilde{X}_{2} \widetilde{X}_{3}\right)-\operatorname{Tr}\left(\widetilde{X}_{2} \widetilde{X}_{1} \widetilde{X}_{3}\right),
$$

where $\widetilde{X}_{i}$ is a preimage of $X_{i}$ in $\mathfrak{g l}_{n}$. It is easily seen that it is skew-symmetric and that the value is independent of the choices of lifts.

LEmma 2.2. There is a unique multiple of the degree 16 equation with integer coefficients which is a nonzero square modulo 4 and is nonzero modulo every odd prime. Furthermore, this equation does not vanish on the trilinear form defined in (2.1) for $n=3$.

We postpone the proof of this fact until the end of the section.

Let $f_{16}(x)$ be this multiple of the equation, and write $f_{16}=f(x)^{2}+4 g(x)$, where $f$ and $g$ are nonzero polynomials with integer coefficients. Introduce a new variable $y$ of degree 8 and consider the equation

$$
y^{2}+f(x) y-g(x)=0 .
$$

This defines a double cover of $\bigwedge^{3} V_{8}$ defined over $\mathbb{Z}$ which is branched along $f_{16}(x)=0$ and which is a reduced scheme modulo any prime $p$.

Let $\mathfrak{e}_{8}$ denote the split Lie algebra of type $\mathrm{E}_{8}$. If we coarsen the root space grading by just taking the coefficient of $\alpha_{2}$ (in Bourbaki notation), we get a $\mathbb{Z}$-graded decomposition $\mathfrak{e}_{8}=$ $\bigoplus_{i=-3}^{3} \mathfrak{g}_{i}$, where

$$
\mathfrak{g}_{-1}=\bigwedge^{3} V_{8}^{*}, \quad \mathfrak{g}_{0}=\mathfrak{g l}\left(V_{8}\right), \quad \mathfrak{g}_{1}=\bigwedge^{3} V_{8}
$$




\section{E. M. RAINS AND S. V SAM}

Proposition 2.4. Let $k$ be a field of characteristic 0 .

(a) There are finitely many orbits of the group $\mathrm{GL}\left(V_{8}\right)$ on both $\mathfrak{g}_{1}$ and $\mathfrak{g}_{-1}$.

(b) There is a bijection between orbit (closures) in $\mathfrak{g}_{1}$ and $\mathfrak{g}_{-1}$. Namely, given an orbit $O \subset \mathfrak{g}_{1}$, send it to the orbit $O^{\prime} \subset \mathfrak{g}_{-1}$, where $O^{\prime}=\left\{x \in \mathfrak{g}_{-1} \mid[x, y]=0\right.$ for all $\left.y \in \mathfrak{g}_{1}\right\}$. If we projectivize $\mathfrak{g}_{1}$ and $\mathfrak{g}_{-1}$, this bijection coincides with projective duality.

(c) Furthermore, if $O^{\prime}$ is the orbit of highest weight vectors, then $O$ has codimension 1.

Proof. Statements (a) and (b) follow from the discussion in [Tev01, § 2.2.A], namely Corollary 2.9 and the text following it. Statement (c) follows from [Tev01, Theorem 9.19].

Proposition 2.5. (a) Pick $\gamma \in \bigwedge^{3} V_{8}$ and a 5-dimensional subspace $U$. Pick a basis $u_{1}, u_{2}, u_{3}$ for $\left(V_{8} / U\right)^{*}$, and let $\gamma^{\prime}=u_{1} \wedge u_{2} \wedge u_{3}$. Then $\left[\gamma, \gamma^{\prime}\right]=0$ if and only if $\gamma \in \bigwedge^{3} U+\bigwedge^{2} U \otimes\left(V_{8} / U\right)$.

(b) The vector $\gamma$ is unstable with respect to $\mathrm{SL}\left(V_{8}\right)$ if and only if there is a 3-dimensional space $V_{3} \subset V_{8}^{*}$ such that $\gamma\left(v_{1}, v_{2},-\right)$ is identically 0 for any $v_{1}, v_{2} \in V_{3}$.

(c) The unstable locus is an irreducible hypersurface and is set-theoretically defined by $f_{16}$.

Proof. (a) Set $W=V_{8} / U$. If $P$ is the parabolic subgroup that preserves $U$, then $\bigwedge^{3} V_{8}$ has a filtration whose quotients are $\bigwedge^{3} U, \bigwedge^{2} U \otimes W, U \otimes \bigwedge^{2} W$, and $\bigwedge^{3} W$. Since the Lie bracket $\mathfrak{g}_{1} \otimes \mathfrak{g}_{-1} \rightarrow \mathfrak{g}_{0}$ is equivariant for GL( $\left(V_{8}\right)$, it follows immediately that if $\gamma^{\prime} \in \bigwedge^{3} W^{*}$ and $\gamma \in \bigwedge^{3} U+$ $\bigwedge^{2} \otimes W$, then $\left[\gamma, \gamma^{\prime}\right]=0$. On the other hand, if we restrict the bracket to various components, we get isomorphisms $\bigwedge^{3} W^{*} \otimes\left(U \otimes \bigwedge^{2} W\right) \rightarrow U \otimes W^{*}$ and $\bigwedge^{3} W^{*} \otimes \bigwedge^{3} W \rightarrow k$. Both $U \otimes W^{*}$ and $k$ are disjoint subspaces in $\mathfrak{g l}\left(V_{8}\right)=\mathfrak{g}_{0}$, so we see that if $\gamma$ has a nonzero component in $U \otimes \bigwedge^{2} W+\bigwedge^{3} W$, then $\left[\gamma, \gamma^{\prime}\right] \neq 0$.

(b) We can rephrase the existence of $V_{3}$ as saying that $\gamma \in \bigwedge^{3} U+\bigwedge^{2} U \otimes\left(V_{8} / U\right)$, where $U=\left(V_{8}^{*} / V_{3}\right)^{*}$. Pick $\gamma$ and assume that $V_{3}$ exists. Pick a basis $e_{1}, \ldots, e_{5}$ for $U$ and extend it to a basis $\left\{e_{1}, \ldots, e_{8}\right\}$ of $V_{8}$. Write $[i j k]$ in place of $e_{i} \wedge e_{j} \wedge e_{k}$. Then $\gamma$ is a sum of trivectors $[i j k]$, where $|\{i, j, k\} \cap\{6,7,8\}| \leqslant 1$. In particular, given the diagonal 1-parameter subgroup $\rho(t)=\left(t^{3}, t^{3}, t^{3}, t^{3}, t^{3}, t^{-5}, t^{-5}, t^{-5}\right)$, we have $\lim _{t \rightarrow 0} \rho(t) \cdot \gamma=0$ (since each $[i j k]$ gets scaled by either $t^{9}$ or $\left.t\right)$, so $\gamma$ is unstable.

In particular, let $\operatorname{Gr}\left(5, V_{8}\right)$ be the Grassmannian of 5 -dimensional subspaces of $V_{8}$. Let $\mathcal{R}_{5}$ be the rank 5 tautological bundle. The trivial bundle $\bigwedge^{3} V_{8}$ has a rank 40 subbundle $\bigwedge^{3} \mathcal{R}_{5}+$ $\bigwedge^{2} \mathcal{R}_{5} \otimes\left(V_{8} / \mathcal{R}_{5}\right)$; call this bundle $\mathcal{E}$. Then $\mathcal{E}$ is the total space of a variety whose image under the projection $\bigwedge^{3} V_{8} \times \operatorname{Gr}\left(5, V_{8}\right) \rightarrow \bigwedge^{3} V_{8}$ is contained in the unstable locus by the previous paragraph. In characteristic 0 , the image has codimension 1 by statement (a) and Proposition 2.4. In particular, the function $n \mapsto \chi\left(\operatorname{Gr}\left(5, V_{8}\right) ; \operatorname{Sym}^{n}\left(\mathcal{E}^{*}\right)\right)$ is a polynomial of degree 55. By flatness, the same is true in any characteristic, so the image has codimension 1 always.

So the image is an irreducible hypersurface, and it is contained in the unstable locus. In characteristic 0 , the unstable locus is an irreducible hypersurface defined by $f_{16}$ because every invariant polynomial is of the form $\lambda f_{16}^{k}$ for $\lambda \in k$. So the two hypersurfaces coincide in this case. In general, let $R$ be a complete discrete valuation ring (DVR) with residue field $k$ and characteristic 0 fraction field. Choose a destabilizing 1-parameter subgroup of $\gamma$ and diagonalize it so that we can lift it to a diagonal subgroup over $R$. In particular, we can choose a lift $\widetilde{\gamma}$ of $\gamma$ over $R$ which is destabilized by this subgroup. The locus of 5 -dimensional subspaces $U$ such that $\widetilde{\gamma} \in \bigwedge^{3} U+\bigwedge^{2} U \otimes\left(V_{8} / U\right)$ is a closed subvariety of $\operatorname{Gr}\left(5, R^{8}\right)$. In particular, there exists such a subspace for $\gamma$ over $k$. This implies that the two hypersurfaces coincide in any characteristic and proves statement (c). 


\section{VECTOR BUNDLES ON GENUS 2 CURVES AND TRIVECTORS}

\subsection{Proof of Lemma 2.2}

Let $\alpha$ denote the form in (2.1).

Lemma 2.6. Let $X \in \mathfrak{p g l}_{n}$. Then $X$ can be lifted to a rank 1 element of $\mathfrak{g l}_{n}$ if and only if the alternating bilinear form $\alpha(X,-,-)$ has rank $2 n-2$.

Proof. Pick any lift $\widetilde{X} \in \mathfrak{g l}_{n}$ of $X$. Since the trace pairing on $\mathfrak{g l}_{n}$ is perfect, it follows that $[A, B]=0$ if and only if $\alpha(A, B, C)=0$ for all $C$. In particular, $\alpha(X,-,-)$ has rank $2 n-2$ if and only if the centralizer of $\widetilde{X}$ in $\mathfrak{g l}_{n}$ has codimension $2 n-2$, if and only if $\widetilde{X}$ has an eigenspace of dimension $n-1$. Subtracting this eigenvalue gives a rank 1 lift of $X$.

Proposition 2.7. For $n \geqslant 3$, the projective stabilizer of the form $[\alpha] \in \mathbb{P}\left(\bigwedge^{3}\left(\mathfrak{p g l}_{n}\right)^{*}\right)$ in $\operatorname{PGL}\left(\mathfrak{p g l}_{n}\right)$ is $\operatorname{Aut}\left(\mathrm{PGL}_{n}\right)$.

Proof. The stabilizer certainly contains $\mathrm{Aut}\left(\mathrm{PGL}_{n}\right)$, so we need simply show that there are no more automorphisms. By Lemma 2.6, it suffices to show this for the automorphism group of the rank 1 locus in $\mathbb{P}\left(\mathfrak{p g l}_{n}\right)$. Since $n \geqslant 3$, an element of $\mathfrak{p g l}_{n}$ with a rank 1 lift has a unique such lift, and thus the rank 1 locus in $\mathbb{P}\left(\mathfrak{g l}_{n}\right)$ embeds in $\mathbb{P}\left(\mathfrak{p g l}_{n}\right)$. Moreover, since the rank 1 locus is isomorphic to $\mathbb{P}\left(V_{n}\right) \times \mathbb{P}\left(V_{n}^{*}\right)$, the pullback of $\mathcal{O}_{\mathbb{P}\left(\mathfrak{g l}_{n}\right)}(1)$ is the unique $n$th root of the anticanonical bundle. It follows that any linear automorphism of the locus in $\mathbb{P}\left(\mathfrak{p g l}_{n}\right)$ lifts to a linear automorphism in $\mathbb{P}\left(\mathfrak{g l}_{n}\right)$ preserving the identity. In other words, the stabilizer of the rank 1 locus in $\mathbb{P}\left(\mathfrak{p g l}_{n}\right)$ is contained in the subgroup of the automorphism group of $\mathbb{P}\left(V_{n}\right) \times \mathbb{P}\left(V_{n}^{*}\right)$ preserving the natural incidence relation. As this is precisely Aut $\left(\mathrm{PGL}_{n}\right)$, the claim follows.

Note that $\mathfrak{p g l}_{n}^{*}$ is canonically identified with $\mathfrak{s l}_{n}$ : In characteristic 0 , we may use the Killing form to identify $\mathfrak{p g l}_{n}$ with its dual (up to an overall scalar). This fails in characteristic dividing $n$, however, in which case there is an $\operatorname{Aut}\left(\mathfrak{p g l}_{n}\right)$-invariant linear functional on $\mathfrak{p g l} \mathfrak{g l}_{n}$ but no Aut $\left(\mathfrak{p g l} \mathfrak{g l}_{n}\right)$ invariant element. Indeed, $\mathfrak{g l}_{n}$ has both an invariant functional $\mathrm{Tr}$ and an invariant element 1 , and since $\operatorname{Tr}(1)=0$ in characteristic dividing $n$, the trace functional survives. We see that in fact the dual of $\mathfrak{p g l}_{n}=\mathfrak{g l}_{n} / 1$ is canonically identified with the annihilator of 1 under the trace pairing, that is, the Lie algebra $\mathfrak{s l}_{n}$ of traceless matrices.

Corollary 2.8. The orbit $\operatorname{GL}\left(\mathfrak{s l}_{3}\right) \alpha$ is dense in $\bigwedge^{3}\left(\mathfrak{s l}_{3}\right)$.

Proof. By Proposition 2.7, the dimension of the orbit is $\operatorname{dim}\left(\mathrm{GL}\left(\mathfrak{s l}_{3}\right)\right)-\operatorname{dim}\left(\operatorname{Aut}\left(\mathrm{PGL}_{3}\right)\right)=$ $64-8=56=\operatorname{dim}\left(\bigwedge^{3}\left(\mathfrak{s l}_{3}\right)\right)$.

For any commutative ring $R$, any element of $R\left[\bigwedge^{3}\left(R^{8}\right)\right]^{\mathrm{SL}_{8}}$ can be evaluated on any triple $\left(V_{8}, \omega, \alpha^{\prime}\right)$ where $V_{8} \cong R^{8}, \omega: R \cong \bigwedge^{8}\left(V_{8}\right)$, and $\alpha^{\prime} \in \bigwedge^{3}\left(V_{8}\right)$. Choose an isomorphism $\omega_{\mathfrak{s l}_{3}}: \mathbb{Z} \cong$ $\bigwedge^{8}\left(\mathfrak{s l}_{3}(\mathbb{Z})\right)$, so that $\left(\mathfrak{s l}_{3}, \omega_{\mathfrak{s l}_{3}}, \alpha_{\mathfrak{s l}_{3}}\right)$ is a triple of the above form. Note that $A \mapsto-A^{T}$ has determinant -1 on $\mathfrak{s l}_{3}$, and thus either choice of $\omega_{\mathfrak{s l}_{3}}$ gives an isomorphic triple.

Now, let $\iota$ be a degree 16 element of $\mathbb{Q}\left[\bigwedge^{3}\left(\mathbb{Q}^{8}\right)\right]^{\mathrm{SL}_{8}}$. By clearing denominators and eliminating content, we may ensure that $\iota$ is a primitive element of $\mathbb{Z}\left[\bigwedge^{3}\left(\mathbb{Z}^{8}\right)\right]^{\mathrm{SL}_{8}}$, and this determines $\iota$ up to a sign. Since $\left(\mathfrak{s l}_{3}, \omega_{\mathfrak{s l}_{3}}, \alpha_{\mathfrak{s l}_{3}}\right)$ projectively generates a dense orbit in all characteristics (Corollary 2.8), the value $\iota\left(\mathfrak{s l}_{3}, \omega_{\mathfrak{s l}_{3}}, \alpha_{\mathfrak{s l}_{3}}\right)$ is nonzero modulo all primes and is thus a unit. We may thus choose the representative of $\iota$ such that $\iota\left(\mathfrak{s l}_{3}, \omega_{\mathfrak{s l}_{3}}, \alpha_{\mathfrak{s l}_{3}}\right)=1$.

Lemma 2.9. A triple $\left(V_{8}, \omega, \alpha\right)$ over an algebraically closed field is equivalent to $\left(\mathfrak{s l}_{3}, \omega_{\mathfrak{s l}_{3}}, \alpha_{\mathfrak{s l}_{3}}\right)$ if and only if $\iota\left(V_{8}, \omega, \alpha\right)=1$. 


\section{E. M. RAINS AND S. V SAM}

Proof. Since triples equivalent to $\left(\mathfrak{s l}_{3}, \omega_{\mathfrak{s l}_{3}}, \alpha_{\mathfrak{s l}_{3}}\right)$ are dense in $\bigwedge^{3}\left(\mathbb{Z}^{8}\right) / / \mathrm{G}_{m}$, every triple with nonzero invariant is equivalent to one of the form $\left(\mathfrak{s l}_{3}, \omega_{\mathfrak{s l}_{3}}, c \alpha_{\mathfrak{s l}_{3}}\right)$ for some $c \neq 0$. It suffices to show that two such triples are equivalent if and only if they have the same invariant. Since the invariant is $c^{16}$, there is nothing to show in characteristic 2, while in odd characteristic we need simply show that the triples with parameter $c$ and $\zeta_{16} c$ are equivalent. Such an equivalence is given by the determinant 1 endomorphism $A \mapsto-\zeta_{16} A^{T}$ of $\mathfrak{s l}_{3}$.

Proposition 2.10. The polynomial $\iota$ is a nonzero square modulo 4.

Proof. Let $Y=\operatorname{Spec}\left(\mathbb{Z}\left[\bigwedge^{3}\left(\mathbb{Z}^{8}\right)\right][1 / \iota]\right)$. For any geometric point of $Y$, the rank 4 locus $Z$ of the corresponding trilinear form is isomorphic to $\mathbb{P}^{2} \times \mathbb{P}^{2}$. There are two isomorphism classes of line bundles on $\mathbb{P}^{2} \times \mathbb{P}^{2}$ with Hilbert polynomial $(k+1)(k+2)^{2}(k+3) / 4$ relative to the ample bundle $\mathcal{O}(1,1)$, and thus the corresponding subscheme of $\operatorname{Pic}(Z / Y)$ is an étale double cover $X$ of $Y$.

Since $Y$ is affine with $\operatorname{Pic}(Y)=1$, the scheme $X$ is affine and has an equation of the form $y^{2}+f y+g=0$, where since $X$ is étale, $f^{2}-4 g$ must be a unit. The unit group is generated by \pm 1 and $\iota$, so $f^{2}-4 g= \pm \iota^{\ell}$ for some integer $\ell$. By clearing denominators, we may ensure that $f$ and $g$ are polynomials, making $\ell \geqslant 0$. If $\ell>1$, then $f$ is congruent $\bmod 2$ to a multiple of $\iota$, and thus we may add a suitable polynomial to $y$ to make $f$ a multiple of $\iota$, and thus $g$ a multiple of $\iota^{2}$, and we may eliminate the common factor.

We thus have an expression for $X$ as $y^{2}+f y+g=0$, where $f^{2}-4 g$ is one of $\{1,-1, \iota,-\iota\}$. Now, consider points of $Y(\mathbf{R})$ equivalent to $\alpha_{\mathfrak{s l}_{3}}$ and $\alpha_{\mathfrak{S u}_{3}}$ (with an arbitrary choice of volume form in the latter case). The first point has $\iota=1$ and has a pair of real preimages in $X$, and thus $f^{2}-4 g$ cannot be -1 or $-\iota$. In contrast, the rank 4 locus of $\alpha_{\mathfrak{s u}_{3}}$ is the restriction of scalars of $\mathbb{P}_{\mathbf{C}}^{2}$, and thus that point has no real preimage, ruling out $f^{2}-4 g=1$. In particular, $\iota=f^{2}-4 g$, which finishes the proof.

Remark 2.11. By taking the integral closure of $\mathbb{Z}\left[\bigwedge^{3}\left(V_{8}\right)\right]$ in $\mathbb{Z}[X]$, we obtain a natural extension of this double cover to all of $\bigwedge^{3}\left(V_{8}\right)$, which again over $\mathbb{Z}[1 / 2]$ is given by $y^{2}=\iota$, and over any field has ramification locus $\iota=0$. In particular, $\iota$ defines an irreducible hypersurface in all characteristics and is reduced in characteristics different from 2.

\section{Generalities on vector bundles on a genus 2 curve}

Some general background for this section can be found in [Pop13].

Let $C$ be a smooth curve of genus 2 over a field $k$, and assume that there is a $k$-rational Weierstrass point $P$. Let $\mathrm{J}^{1}(C)$ denote the space of line bundles of degree 1 over $C$, which has a natural divisor $\Theta$ given by $\left\{\mathcal{L} \mid \mathrm{H}^{0}(C ; \mathcal{L}) \neq 0\right\}$. Let $\mathrm{J}(C)=\mathrm{J}^{0}(C)$ be the Jacobian of $C$, that is, the space of line bundles of degree 0 over $C$.

Let $\mathrm{SU}_{3}(C)$ be the coarse moduli space of rank 3 semistable vector bundles on $C$ modulo S-equivalence [Pop13, $\S 2.5]$. Let $\mathrm{SU}_{3}^{\mathrm{s}}(C)$ be the subscheme of stable vector bundles in $\mathrm{SU}_{3}(C)$. Let $\Omega_{\mathrm{SU}_{3}(C)}^{1}$ denote the cotangent sheaf of $\mathrm{SU}_{3}(C)$. On $\mathrm{SU}_{3}^{\mathrm{s}}(C)$, the fiber of $\Omega_{\mathrm{SU}_{3}(C)}^{1}$ over $\mathcal{E}$ is $\mathrm{H}^{0}\left(C ; \mathfrak{p g l}(\mathcal{E}) \otimes \omega_{C}\right)($ see $[$ HL10, Theorem 4.5.4]).

Given a vector bundle $\mathcal{E}$ over $C$, define

$$
\Theta_{\mathcal{E}}=\left\{\mathcal{L} \in \mathrm{J}^{1}(C) \mid \mathrm{H}^{0}(C ; \mathcal{E} \otimes \mathcal{L}) \neq 0\right\} .
$$

LEMma 3.1. If $\mathcal{E}$ is a semistable rank 3 vector bundle with trivial determinant, then $\Theta_{\mathcal{E}}$ is a divisor in $\mathrm{J}^{1}(C)$ which is linearly equivalent to $3 \Theta$. 


\section{VECTOR BUNDLES ON GENUS 2 CURVES AND TRIVECTORS}

Proof. By the Riemann-Roch theorem, $\chi(\mathcal{E} \otimes \mathcal{L})=0$, so $\Theta_{\mathcal{E}}$ is the zero locus of a determinant (see, for example, [Pop13, §3]) and hence has codimension at most 1. By [Ray82, Corollaire 1.7.4], there exists an $\mathcal{L} \in \mathrm{J}^{1}(C)$ with $\mathrm{H}^{0}(C ; \mathcal{E} \otimes \mathcal{L})=0$, so the codimension of $\Theta_{\mathcal{E}}$ is exactly 1 .

So, the locus

$$
\left\{(E, \mathcal{L}) \in \mathrm{SU}_{3}(C) \times \mathrm{J}^{1}(C) \mid \mathrm{H}^{0}(C ; \mathcal{E} \otimes \mathcal{L}) \neq 0\right\}
$$

is a divisor in $\mathrm{SU}_{3}(C) \times \mathrm{J}^{1}(C)$ and hence is the zero section of some line bundle whose fibers over a fixed point $[\mathcal{E}]$ of $\mathrm{SU}_{3}(C)$ are the line bundles cutting out $\Theta_{\mathcal{\varepsilon}}$. Thus, we see that these line bundles are algebraically equivalent to one another, and hence we get a map $f: \mathrm{SU}_{3}(C) \rightarrow$ $\operatorname{Pic}\left(\mathrm{J}^{1}(C)\right) \cong \operatorname{Jac}(C)$. In the special case $\mathcal{E}=\mathcal{L}_{1} \oplus \mathcal{L}_{2} \oplus \mathcal{L}_{3}$ for line bundles $\mathcal{L}_{i}$ (which must be of degree 0 by semistability), we see that $\Theta_{\mathcal{E}}$ is linearly equivalent to $\Theta_{\mathcal{L}_{1}}+\Theta_{\mathcal{L}_{2}}+\Theta_{\mathcal{L}_{3}}$, which in turn is linearly equivalent to $3 \Theta$ via the theorem of the square.

We see that $f$ contracts positive-dimensional subvarieties (in particular, curves). Now let $\mathcal{M}$ be an ample line bundle on $\operatorname{Jac}(C)$. Then $f^{*}(\mathcal{M})$ is constant along such curves and hence cannot be ample. Finally, the Picard group of $\mathrm{SU}_{3}(C)$ is isomorphic to $\mathbb{Z}$ with ample generator [Hof12, Corollary 3.4], so we conclude that $f^{*}(\mathcal{M})$ is trivial, which implies that $f$ is a constant map. Hence, $\Theta_{\mathcal{E}}$ is linearly equivalent to $3 \Theta$ for all $\mathcal{E}$.

Lemma 3.1 implies that there is a well-defined morphism

$$
\theta: \mathrm{SU}_{3}(C) \rightarrow|3 \Theta| \cong \mathbb{P}^{8}, \quad \mathcal{\varepsilon} \mapsto \Theta_{\mathcal{\varepsilon}} .
$$

Define $\mathcal{O}_{\mathrm{SU}_{3}(C)}(1)=\theta^{*} \mathcal{O}_{\mathbb{P}^{8}}(1)$. Let $\iota$ be the hyperelliptic involution of $C$.

Proposition 3.2. The morphism $\theta$ commutes with the involution $\mathcal{E} \mapsto \iota^{*} \mathcal{E}^{*}$ on $\mathrm{SU}_{3}(C)$, and this involution is not the identity map on $\mathrm{SU}_{3}(C)$.

Proof. We may assume that $k$ is algebraically closed. The first statement is [Ort05, Proposition 4.1]. For the second statement, we will adapt the argument in [Ort05, §4], which uses results only stated in characteristic 0 .

Let $\mathcal{L}$ be a line bundle on $C$ of degree 1 with $\iota^{*} \mathcal{L} \cong \mathcal{L}$, and let $\mathrm{SU}_{2}(C ; \mathcal{L})$ be the moduli space of isomorphism classes of stable rank 2 vector bundles with determinant $\mathcal{L}$. As in [Ort05, $\S 4$ ], it is sufficient to find $\mathcal{F} \in \mathrm{SU}_{2}(C ; \mathcal{L})$ such that $\iota^{*} \mathcal{F} \not \mathcal{F} \otimes \alpha$ for every $\alpha \in \mathrm{J}(C)[2]$.

First, $\mathrm{SU}_{2}(C ; \mathcal{L})$ can be embedded into $\mathbb{P}^{5}$ as the complete intersection of two quadrics (see [DR76, Theorem 1] if the characteristic is different from 2, and [Bho90] for characteristic 2) and its Picard group is isomorphic to $\mathbb{Z}$ [Hof12, Corollary 3.4]. Furthermore, only one of the generators of its Picard group has global sections, and so any automorphism of $\mathrm{SU}_{2}(C ; \mathcal{L})$ is trivial on its Picard group. Since $\mathrm{SU}_{2}(C ; \mathcal{L})$ is linearly normal in $\mathbb{P}^{5}$, any automorphism of $\mathrm{SU}_{2}(C ; \mathcal{L})$ extends to an automorphism of $\mathbb{P}^{5}$. We finish by using the proof of [New68, Lemma 7].

Proposition 3.3. The line bundle $\mathcal{O}_{\mathrm{SU}_{3}(C)}(1)$ generates the Picard group of $\mathrm{SU}_{3}(C)$, which is isomorphic to $\mathbb{Z}$. Furthermore, $\mathrm{SU}_{3}(C)$ is a normal, Gorenstein, locally factorial, projective, geometrically irreducible variety, and its canonical bundle is $\mathcal{O}_{\mathrm{SU}_{3}(C)}(-6)$.

Proof. The fact that $\mathrm{SU}_{3}(C)$ is a locally factorial projective variety is [Hof12, Corollary 3.8] and $\operatorname{Pic}\left(\mathrm{SU}_{3}(C)\right) \cong \mathbb{Z}$ is [Hof12, Corollary 3.4]. The normal and Gorenstein properties are shown in $[\mathrm{VM} 08, \S 2]$. The calculation of the canonical bundle was done in characteristic 0 in $[\mathrm{DN} 89, \S 7.5]$, but subject to the other properties above being known, the same proof goes through.

Proposition 3.4. The morphism $\theta$ is surjective, finite, flat and has degree 2. It is branched along a degree 6 hypersurface in $|3 \Theta| \cong \mathbb{P}^{8}$. 


\section{E. M. RAINS AND S. V SAM}

Proof. We may assume that $k$ is algebraically closed. Let $R$ be a complete DVR whose residue field is $k$ and whose fraction field $\mathrm{K}$ is of characteristic 0 . Then there exists a smooth curve $\mathcal{C}$ over $R$ such that $\mathcal{C}_{k}=C$ and $\mathcal{C}_{\mathrm{K}}$ is smooth.

From [Lan04, Theorem 4.1], there is a projective scheme $M_{\mathcal{C} / R}(r, d)$ of finite type over $R$ such that for any $R$-scheme $T$, the set $\operatorname{Hom}\left(T, M_{\mathcal{C} / R}(r, d)\right)$ is the set of S-equivalence classes of families of rank $r$ semistable bundles of degree $d$ on the geometric fibers of $T \times{ }_{R} \mathcal{C} \rightarrow T$ which are flat over $T$. Consequently, given $\mathcal{E}_{\mathrm{K}} \in \mathrm{SU}_{3}\left(\mathcal{C}_{\mathrm{K}}\right)$, we can extend it uniquely to a flat bundle $\mathcal{E}_{R}$ over $\mathcal{C}_{R}$ and then reduce it to a bundle $\mathcal{E}_{k} \in \mathrm{SU}_{3}(C)$. This gives a commutative square

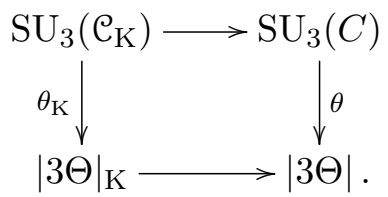

We know that $\theta_{\mathrm{K}}$ is a surjective degree 2 morphism [Las96, $\S \mathrm{V}$, Lemma 5]. The bottom map is surjective onto $k$-points, and if we pick a preimage in $|3 \Theta|_{K}$ of each point in $|3 \Theta|$, then the restriction of the composition $\mathrm{SU}_{3}\left(\mathcal{C}_{\mathrm{K}}\right) \rightarrow|3 \Theta|_{\mathrm{K}} \rightarrow|3 \Theta|$ to points that lie over these preimages is surjective and generically of degree 2 . We deduce that $\theta$ is surjective and of degree at most 2 . To get that the degree is 2, we use Proposition 3.2.

Since $\mathrm{SU}_{3}(C)$ is Cohen-Macaulay (Proposition 3.3 gives Gorenstein), $\theta$ is a finite flat morphism and so $\theta_{*} \mathcal{O}_{\mathrm{SU}_{3}(C)}$ is a rank 2 vector bundle on $\mathbb{P}^{8}$. Furthermore, $\omega_{\mathrm{SU}_{3}(C)} \cong \theta^{*} \omega_{\mathbb{P}^{8}} \otimes \mathcal{R}$, where the ramification divisor is a section of $\mathcal{R}$. By Proposition 3.3, we conclude that $\mathcal{R}=\mathcal{O}_{\mathrm{SU}_{3}(C)}(3)$, and so $\theta_{*} \mathcal{O}_{\mathrm{SU}_{3}(C)} \cong \mathcal{O}_{\mathbb{P}^{8}} \oplus \mathcal{O}_{\mathbb{P}^{8}}(-3)$. We conclude that $\theta$ is branched along a degree 6 hypersurface.

Corollary 3.5. The Verlinde formula is valid for $\mathrm{SU}_{3}(C)$ in arbitrary characteristic. More precisely, $\mathcal{O}_{\mathrm{SU}_{3}(C)}(d)$ has cohomology concentrated in a single degree $i$, with $i=0$ if $d \geqslant 0$ and $i=8$ if $d \leqslant-6$, and the cohomology vanishes otherwise. The Hilbert polynomial of $\mathcal{O}_{\mathrm{SU}_{3}(C)}(d)$ is

$$
\chi\left(\mathcal{O}_{\mathrm{SU}_{3}(C)}(d)\right)=\frac{2}{8 !}(d+1)(d+2)(d+3)^{2}(d+4)(d+5)\left(d^{2}+6 d+56\right) .
$$

Proof. We can embed $\mathrm{SU}_{3}(C)$ as a degree 6 hypersurface in a weighted projective space $\mathbb{P}$ with 9 variables of degree 1 and 1 variable of degree 3 . So it has a locally free resolution $0 \rightarrow \mathcal{O}_{\mathbb{P}}(-6) \rightarrow \mathcal{O}_{\mathbb{P}} \rightarrow \mathcal{O}_{\mathrm{SU}_{3}(C)} \rightarrow 0$, and the cohomology of $\mathcal{O}_{\mathbb{P}}(d)$ is at most in a single degree (either 0 or 9 ). Hence, the cohomology of $\mathcal{O}_{\mathrm{SU}_{3}(C)}(d)$ is at most in a single degree (either in 0 or 8). So its Hilbert polynomial is independent of the characteristic and can be read off from the locally free resolution.

\section{Discriminants}

As in the previous section, let $C$ be a smooth genus 2 curve over $k$, and let $P \in C(k)$ be a Weierstrass point. Let $\mathrm{J}^{1}(C)$ be the space of degree 1 line bundles over $C$ with its divisor $\Theta$ from above. From Proposition 3.4, we have a degree 2 map

$$
\theta: \mathrm{SU}_{3}(C) \rightarrow|3 \Theta| \cong \mathbb{P}^{8}
$$

which is branched along a degree 6 hypersurface in $\mathbb{P}^{8}$. Let $\left(\mathbb{P}^{8}\right)^{\mathrm{s}}$ be the image of the stable locus in $\mathrm{SU}_{3}(C)$ under $\theta$. 


\section{VECTOR BUNDLES ON GENUS 2 CURVES AND TRIVECTORS}

Using (2.1), we have maps

$$
\gamma_{\varepsilon}: \bigwedge^{3} \mathrm{H}^{0}\left(C ; \mathfrak{p g l}(\mathcal{E}) \otimes \omega_{C}\right) \rightarrow \mathrm{H}^{0}\left(C ; \omega_{C}^{\otimes 3}\right) \rightarrow \mathrm{H}^{0}\left(P ; \omega_{C}^{\otimes 3}\right) \cong k,
$$

where the last isomorphism is well defined up to a global choice (independent of $\mathcal{E}$ ) of scalar. This gives a map

$$
\theta^{*} \Omega_{\left(\mathbb{P}^{8}\right)^{\mathrm{s}}}^{3} \rightarrow \Omega_{\mathrm{SU}_{3}^{\mathrm{s}}(C)}^{3} \stackrel{\gamma}{\rightarrow} \mathcal{O}_{\mathrm{SU}_{3}^{\mathrm{s}}(C)}=\theta^{*} \mathcal{O}_{\left(\mathbb{P}^{8}\right)^{\mathrm{s}}} .
$$

LEMma 4.1. The composition descends as an anti-symmetrized form on $\left(\mathbb{P}^{8}\right)^{\mathrm{s}}$, so it gives a section of $\left(\bigwedge^{3} \mathcal{T}_{\left(\mathbb{P}^{8}\right)^{\mathrm{s}}}\right)(-3)$, where $\mathcal{T}$ denotes the tangent sheaf.

Proof. The involution $\mathcal{E} \mapsto \iota^{*} \mathcal{E}^{*}$ induces an involution on $\theta_{*} \mathcal{O}_{\mathrm{SU}_{3}(C)}$ which we denote by $\iota$. We have an exact sequence

$$
0 \rightarrow \mathcal{O}_{\mathbb{P} 8} \rightarrow \theta_{*} \mathcal{O}_{\mathrm{SU}_{3}(C)} \stackrel{1-\iota}{\longrightarrow} \theta_{*} \mathcal{O}_{\mathrm{SU}_{3}(C)},
$$

which shows that the quotient $\theta_{*} \mathcal{O}_{\mathrm{SU}_{3}(C)} / \mathcal{O}_{\mathbb{P}^{8}} \cong \mathcal{O}_{\mathbb{P}^{8}}(-3)$ is the image of the map $1-\iota$.

First, suppose that the characteristic of $k$ is different from 2. Then $\iota$ acts as -1 on $\gamma$ since $\mathcal{E} \mapsto \iota^{*} \mathcal{E}^{*}$ acts contravariantly on maps and, in particular, $\gamma$ is in the image of $1-\iota$. If the characteristic of $k$ is 2 , then we can write $\gamma_{\mathcal{E}}$ as the difference of the two forms $\left(X_{1}, X_{2}, X_{3}\right) \mapsto$ $\operatorname{Tr}\left(\widetilde{X}_{1} \widetilde{X}_{2} \widetilde{X}_{3}\right)$ and $\left(X_{1}, X_{2}, X_{3}\right) \mapsto \operatorname{Tr}\left(\widetilde{X}_{2} \widetilde{X}_{1} \widetilde{X}_{3}\right)=\operatorname{Tr}\left(\widetilde{X}_{3} \widetilde{X}_{2} \widetilde{X}_{1}\right)$, where we have chosen lifts $\widetilde{X}_{i} \in \mathrm{H}^{0}\left(C ; \mathfrak{g l}(\mathcal{E}) \otimes \omega_{C}\right)$ with trace 0 to make it well defined. These two forms are swapped under the involution on $\mathrm{SU}_{3}(C)$, so again, $\gamma$ is in the image of $1-\iota$.

So $\gamma$ descends to a form $\Omega_{\left(\mathbb{P}^{8}\right)^{\mathrm{s}}}^{3} \rightarrow \mathcal{O}_{\left(\mathbb{P}^{8}\right)^{\mathrm{s}}}(-3)$, that is, a section of $\left(\bigwedge^{3} \mathcal{T}_{\left(\mathbb{P}^{8}\right)^{\mathrm{s}}}\right)(-3)$.

The complement of the stable locus in $\mathrm{SU}_{3}(C)$ has codimension 3, and so its image in $\mathbb{P}^{8}$ has codimension at least 3 . In particular, given a vector bundle on $\mathbb{P}^{8}$, any section of it over $\left(\mathbb{P}^{8}\right)^{\mathrm{S}}$ extends to a section on all of $\mathbb{P}^{8}$. So we get a section of $\left(\bigwedge^{3} \mathcal{T}_{\mathbb{P}^{8}}\right)(-3)$. Finally, $\mathrm{H}^{0}\left(\mathbb{P}^{8} ;\left(\bigwedge^{3} \mathcal{T}_{\mathbb{P}^{8}}\right)(-3)\right)=\bigwedge^{3}\left(\mathrm{H}^{0}\left(\mathbb{P}^{8} ; \mathcal{O}(1)\right)^{*}\right)$, so we get

$$
\gamma_{(C, P)} \in \bigwedge^{3}\left(\mathrm{H}^{0}\left(\mathrm{SU}_{3}(C) ; \Theta\right)^{*}\right) \text {. }
$$

Definition 4.2. The fiber of $\left(\bigwedge^{3} \mathcal{T}_{\mathbb{P}^{8}}\right)(-3)$ over a point of $\mathbb{P}^{8}$ is of the form $\bigwedge^{3}\left(k^{8}\right)$; in Section 2 , we identified a $\mathrm{GL}_{8}$-invariant hypersurface in $\Lambda^{3}\left(k^{8}\right)$, which we called the hyperdiscriminant. Given a section $\gamma \in \mathrm{H}^{0}\left(\mathbb{P}^{8} ;\left(\bigwedge^{3} \mathcal{T}_{\mathbb{P}^{8}}\right)(-3)\right)$, let $D_{\gamma}$ be the subscheme of points where the corresponding element in the fiber has vanishing hyperdiscriminant.

Locally, the hyperdiscriminant is a degree 16 equation, and in basis-independent terms, the line that it spans is given by $\left(\operatorname{det} k^{8}\right)^{6} \subset \operatorname{Sym}^{16}\left(\Lambda^{3}\left(k^{8}\right)\right)$. So the equation defining $D_{\gamma}$ is a section of $\left(\operatorname{det} \mathcal{T}_{\mathbb{P}^{8}}\right)^{6}(-48)=\mathcal{O}_{\mathbb{P}^{8}}(6)$. As an aside, we can interpret $\mathbb{P}^{8}$ as parametrizing lines in a 9 -dimensional vector space, so it has a tautological rank 8 quotient bundle $\mathcal{Q}$, and $\mathcal{T}_{\mathbb{P} 8} \cong$ $\mathcal{Q} \otimes \mathcal{O}_{\mathbb{P}^{8}}(1)$.

The following two statements are the main results about $\gamma_{(C, P)}$ for this section. Their proofs are given below in separate subsections.

Proposition 4.3. The scheme $D_{\gamma_{(C, P)}}$ is the branch locus of $\theta$.

Proposition 4.4. Pick a basis for $\mathrm{H}^{0}\left(\mathrm{SU}_{3}(C) ; \Theta\right)^{*}$. Then $\gamma_{(C, P)} \in \bigwedge^{3} k^{9}$ is a stable element with respect to the natural action of $\mathrm{SL}_{9}(k)$. 


\section{E. M. RAINS AND S. V SAM}

\subsection{Proof of Proposition 4.3}

LEMMA 4.5. Let $C$ be a hyperelliptic curve over an algebraically closed field with hyperelliptic involution $\iota$, and let $L$ be a line bundle such that $\iota^{*} L \cong L$ and $\chi(L)=0$. Then there is a lift $(\widetilde{C}, \widetilde{L})$ of this pair to characteristic 0 such that $\mathrm{h}^{0}(\widetilde{L})=\mathrm{h}^{0}(L)$.

Proof. If $\mathrm{h}^{0}(L)=0$, any lift of $L$ satisfies the condition. Now, suppose $\mathrm{h}^{0}(L)=1$. Then $L$ is represented by a unique effective divisor, which must therefore be $\iota$-invariant. If the support of this divisor contains a non-Weierstrass point, or a Weierstrass point with multiplicity greater than 1 , then we can replace that subdivisor by any representative of the hyperelliptic class $\eta$, contradicting the uniqueness. So $L$ is uniquely represented by a multiplicity-free sum of Weierstrass points; since Weierstrass points lift to characteristic 0 , it follows that $L$ lifts as well.

Now, suppose $d=\mathrm{h}^{0}(L)-1>0$. Since $\pi: C \rightarrow \mathbb{P}^{1}$ is flat and 0-dimensional, $\pi_{*} L$ is a rank 2 vector bundle on $\mathbb{P}^{1}$ and has the same cohomology as $L$. Since $\chi(L)=0$, we also have $\chi\left(\pi_{*} L\right)=0$, so it follows that

$$
\pi_{*} L \cong \mathcal{O}_{\mathbb{P}^{1}}(d) \oplus \mathcal{O}_{\mathbb{P}^{1}}(-d-2) .
$$

We then find immediately that $\eta^{-d} \otimes L$ is an $\iota$-invariant line bundle with $\mathrm{h}^{0}\left(\eta^{-d} \otimes L\right)=1$, so admits a lift $\widetilde{L}^{\prime}$. We then find that $\widetilde{L}:=\eta^{d} \otimes \widetilde{L}^{\prime}$ is a lift of $L$ with at least $d+1$ global sections, so precisely $d+1$ global sections by semicontinuity.

Lemma 4.6. Let $\mathcal{E}$ be a vector bundle on a hyperelliptic curve $C$ of genus $g$ with $\operatorname{det} \mathcal{E} \cong \mathcal{O}_{C}$. Then for any theta-characteristic $\theta$ ( that is, $\theta^{2} \cong \omega_{C}$ ), we have

$$
\mathrm{h}^{0}\left(C ; \mathcal{E} \otimes \iota^{*} \mathcal{E} \otimes \theta\right) \geqslant \operatorname{rank}(\mathcal{E}) \cdot \mathrm{h}^{0}(C ; \theta) .
$$

Proof. First, assume that the characteristic is different from 2 . Note that $\mathcal{E} \otimes \iota^{*} \mathcal{E} \otimes \theta$ has the structure of an $\iota$-equivariant sheaf (in two ways, depending on a choice of $\iota$-equivariant structure on $\theta$ ). The eigenspaces on the fibers over Weierstrass points of the natural equivariant structure on $\mathcal{E} \otimes \iota^{*} \mathcal{E}$ are the symmetric and anti-symmetric forms, and this will remain true after twisting by $\theta$, except that the eigenspaces will swap at $g+1-\mathrm{h}^{0}(C ; \theta)$ Weierstrass points (for one of the two equivariant structures on $\theta$ ).

Let $\pi: C \rightarrow \mathbb{P}^{1}$ be the double covering map, and let $\pi^{*} \mathcal{V}$ be the largest equivariant subsheaf of $\mathcal{E} \otimes \iota^{*} \mathcal{E} \otimes \theta$ that descends to $\mathbb{P}^{1}$; the sheaf $\pi^{*} \mathcal{V}$ is the kernel of the map to the (-1)-eigenspaces of the fibers at the Weierstrass points. Since $\chi\left(\mathcal{E} \otimes \iota^{*} \mathcal{E} \otimes \theta\right)=0$, we have

$$
\begin{aligned}
\chi\left(\pi^{*} V\right) & =-\left(g+1-2 \mathrm{~h}^{0}(C ; \theta)\right) \frac{r(r+1)}{2}-\left(g+1+2 \mathrm{~h}^{0}(C ; \theta)\right) \frac{r(r-1)}{2} \\
& =-(g+1) r^{2}+2 \mathrm{~h}^{0}(C ; \theta) r,
\end{aligned}
$$

and thus

$$
\operatorname{deg}(\mathcal{V})=\operatorname{deg}\left(\pi^{*} \mathcal{V}\right) / 2=-r^{2}+\mathrm{h}^{0}(C ; \theta) r .
$$

But then the Riemann-Roch theorem gives $\chi(\mathcal{V})=\mathrm{h}^{0}(C ; \theta) r$, so that $\mathcal{V}$, thus $\pi^{*} \mathcal{V}$, has at least that many global sections.

Now, we handle the case that $k$ has characteristic 2 . We may assume as well that $k$ is algebraically closed. By Lemma 4.5 , we can lift $(C, \theta)$ to a pair $(\widetilde{C}, \widetilde{\theta})$ in characteristic 0 satisfying $\mathrm{h}^{0}(\theta)=\mathrm{h}^{0}(\widetilde{\theta})$. Let $\widetilde{\mathcal{E}}$ be any lift of $\mathcal{E}$ to $\widetilde{C}$. Then

$$
\mathrm{h}^{0}\left(C ; \mathcal{E} \otimes \iota^{*} \mathcal{E} \otimes \theta\right) \geqslant \mathrm{h}^{0}\left(\widetilde{C} ; \widetilde{\mathcal{E}} \otimes \iota^{*} \widetilde{\mathcal{E}} \otimes \widetilde{\theta}\right) \geqslant \operatorname{rank}(\widetilde{\mathcal{E}}) \cdot \mathrm{h}^{0}(\widetilde{C} ; \widetilde{\theta})=\operatorname{rank}(\mathcal{E}) \cdot \mathrm{h}^{0}(C ; \theta) .
$$

Corollary 4.7. If $\mathcal{E}$ is a stable rank 3 vector bundle on $C$ with trivial determinant, then $\mathfrak{p g l}(\mathcal{E}) \otimes \mathcal{O}(P)$ has a nonzero global section if and only if $\mathcal{E} \cong \iota^{*} \mathcal{E}^{*}$. 


\section{VECTOR BUNDLES ON GENUS 2 CURVES AND TRIVECTORS}

Proof. Note that $\chi(\mathfrak{p g l}(\mathcal{E}) \otimes \mathcal{O}(P))=0$, so by Serre duality and the Riemann-Roch theorem, $\mathfrak{p g l}(\mathcal{E}) \otimes \mathcal{O}(P)$ has a nonzero global section if and only if the same is true for $\mathfrak{s l}(\mathcal{E}) \otimes \mathcal{O}(P)$, and we will work with the latter condition. Since $\mathcal{E}$ is stable, $\mathrm{h}^{0}(C ; \mathfrak{g l}(\mathcal{E}))=1$, and $\mathrm{H}^{0}(C ; \mathfrak{g l}(\mathcal{E}))$ is spanned by the identity map $\mathcal{E} \rightarrow \mathcal{E}$. In particular, this has nonzero image under the trace map $\mathfrak{g l}(\mathcal{E}) \rightarrow \mathcal{O}_{C}$, so $\mathrm{H}^{0}(C ; \mathfrak{s l}(\mathcal{E}))=0$.

Let $\mathscr{E}$ be a universal sheaf on $C \times \mathrm{SU}_{3}^{\mathrm{s}}(C)$ with projection maps $p$ and $q$. Apply $q_{*}$ to

$$
\left.0 \rightarrow \mathfrak{s l}(\mathscr{E}) \rightarrow \mathfrak{s l}(\mathscr{E}) \otimes p^{*} \mathcal{O}(P) \rightarrow \mathfrak{s l}(\mathscr{E}) \otimes p^{*} \mathcal{O}(P)\right|_{P} \rightarrow 0
$$

to get the 4 -term exact sequence $\left(q_{*} \mathfrak{s l}(\mathscr{E})=0\right.$ by what we just argued)

$$
\begin{aligned}
0 & \rightarrow q_{*}\left(\mathfrak{s l}(\mathscr{E}) \otimes p^{*} \mathcal{O}(P)\right) \rightarrow q_{*}\left(\left.\mathfrak{s l}(\mathscr{E}) \otimes p^{*} \mathcal{O}(P)\right|_{P}\right) \stackrel{\alpha}{\rightarrow} \mathrm{R}^{1} q_{*}(\mathfrak{s l}(\mathscr{E})) \\
& \rightarrow \mathrm{R}^{1} q_{*}\left(\mathfrak{s l}(\mathscr{E}) \otimes p^{*} \mathcal{O}(P)\right) \rightarrow 0 .
\end{aligned}
$$

The locus of interest, that is, stable bundles $\mathcal{E}$ where $\mathrm{H}^{0}(C ; \mathfrak{s l}(\mathcal{E}) \otimes \mathcal{O}(P)) \neq 0$, is set-theoretically defined by $\operatorname{det}(\alpha)$. Let $\mathcal{F}$ and $\mathcal{G}$ denote the source and target of $\alpha$, respectively. So $\operatorname{det}(\alpha)$ is a section of $\operatorname{det}(\mathcal{G}) \otimes \operatorname{det}\left(\mathcal{F}^{*}\right)$. Since $\mathcal{G}=\mathcal{T}_{\mathrm{SU}_{3}^{\mathrm{s}}(C)}$, its determinant is $\mathcal{O}_{\mathrm{SU}_{3}^{\mathrm{s}}(C)}(6)$.

We claim that $\operatorname{det}(\mathcal{F})=\mathcal{O}_{\mathrm{SU}_{3}^{\mathrm{s}}(C)}$. Define $s: \mathrm{SU}_{3}^{\mathrm{s}}(C) \rightarrow C \times \mathrm{SU}_{3}^{\mathrm{s}}(C)$ by $s(\mathcal{E})=(P, \mathcal{E})$. For any sheaf $\mathcal{M}$ on $C \times \mathrm{SU}_{3}^{\mathrm{s}}(C)$, we have $q_{*}\left(\left.\mathcal{M}\right|_{P}\right)=s^{*} \mathcal{M}$. Applying this to $\mathcal{M}=\mathfrak{s l}(\mathscr{E}) \otimes p^{*} \mathcal{O}(P)$, we get

$$
\mathcal{F}=q_{*}\left(\left.\mathfrak{s l}(\mathscr{E}) \otimes p^{*} \mathcal{O}(P)\right|_{P}\right)=s^{*}(\mathfrak{s l}(\mathscr{E})) \otimes s^{*} p^{*} \mathcal{O}(P) .
$$

Note that $s^{*} p^{*} \mathcal{O}(P)$ is trivial, since $p^{*} \mathcal{O}(P) \cong \mathcal{O}$ (image $s$ ) is the line bundle associated with a section of a product. Also, $s^{*}(\mathfrak{s l}(\mathscr{E})) \cong \mathfrak{s l}\left(s^{*} \mathscr{E}\right)$, and $\mathfrak{s l}$ of any vector bundle has trivial determinant. So $\operatorname{det}(\mathcal{F})=\mathcal{O}_{\mathrm{SU}_{3}^{\mathrm{s}}(C)}$.

So $\alpha$ is a section of $\mathcal{O}_{\mathrm{SU}_{3}^{\mathrm{s}}(C)}(6)$. We have an exact sequence

$$
0 \rightarrow \mathrm{H}^{0}(C ; \mathfrak{s l}(\mathcal{E}) \otimes \mathcal{O}(P)) \rightarrow \mathrm{H}^{0}(C ; \mathfrak{g l}(\mathcal{E}) \otimes \mathcal{O}(P)) \rightarrow \mathrm{H}^{0}(C ; \mathcal{O}(P)) .
$$

The third term has dimension 1 , and by Lemma 4.6 with $\eta=\mathcal{O}(P)$, the second term has dimension at least 3 when $\mathcal{E} \cong \iota^{*} \mathcal{E}^{*}$. So $\mathrm{h}^{0}(C ; \mathfrak{s l}(\mathcal{E}) \otimes \mathcal{O}(P)) \geqslant 2$ when $\mathcal{E} \cong \iota^{*} \mathcal{E}^{*}$. The ramification locus is branched along a sextic in $\mathbb{P}^{8}$, so it is a zero section of $\mathcal{O}_{\mathrm{SU}_{3}^{\mathrm{s}}(C)}(3)$. So $\operatorname{det}(\alpha)$ contains the ramification locus with multiplicity either 1 or 2 . But on the ramification locus, the rank of $\alpha$ drops by at least 2 , so the multiplicity is at least 2 . So we conclude that $\mathrm{H}^{0}(C ; \mathfrak{s l}(\mathcal{E}) \otimes \mathcal{O}(P)) \neq 0$ if and only if $\mathcal{E} \cong \iota^{*} \mathcal{E}^{*}$.

Proof of Proposition 4.3. Let $\mathcal{E}$ be a rank 3 stable bundle with trivial determinant. The form $\gamma_{\mathcal{E}}$ may be defined as the composition of either of two maps in the following commutative square:

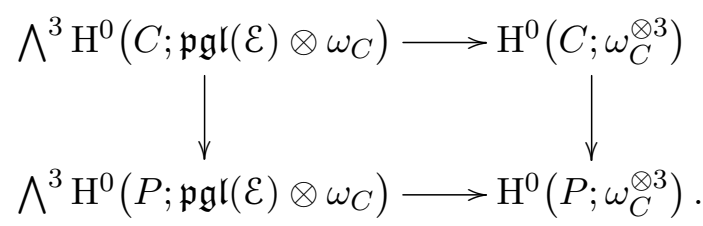

In particular, $\gamma_{\mathcal{E}}$ has nonzero hyperdiscriminant if and only if the restriction

$$
\mathrm{H}^{0}\left(C ; \mathfrak{p g l}(\varepsilon) \otimes \omega_{C}\right) \rightarrow \mathrm{H}^{0}\left(P ; \mathfrak{p g l}(\varepsilon) \otimes \omega_{C}\right)
$$

is an isomorphism. By considering the short exact sequence

$$
\left.0 \rightarrow \mathfrak{p g l}(\mathcal{E}) \otimes \mathcal{O}(P) \rightarrow \mathfrak{p g l}(\mathcal{E}) \otimes \omega_{C} \rightarrow\left(\mathfrak{p g l}(\mathcal{E}) \otimes \omega_{C}\right)\right|_{P} \rightarrow 0,
$$

we see that $\mathrm{H}^{0}\left(C ; \mathfrak{p g l l}(\mathcal{E}) \otimes \omega_{C}\right) \rightarrow \mathrm{H}^{0}\left(P ; \mathfrak{p g l}(\mathcal{E}) \otimes \omega_{C}\right)$ is an isomorphism if and only if $\mathrm{H}^{0}(C ; \mathfrak{p g l}(\mathcal{E})$ $\otimes \mathcal{O}(P))=0($ since $\chi(\mathfrak{p g l}(\mathcal{E}) \otimes \mathcal{O}(P))=0)$. By Corollary 4.7, this is equivalent to $\mathcal{E} \approx \iota^{*} \mathcal{E}^{*}$. 


\section{E. M. RAINS AND S. V SAM}

Putting this together, we see that $\gamma_{\mathcal{E}}$ has nonzero hyperdiscriminant if and only if $\mathcal{E} \neq \iota^{*} \mathcal{E}^{*}$. So by Proposition 3.2, the scheme $D_{\gamma}$ and the branch locus of $\theta$ are the same as sets. Both of them are degree 6 hypersurfaces, and we will show in Corollary 5.3 that $D_{\gamma}$ is irreducible. In particular, they must be equal as schemes.

\subsection{Proof of Proposition 4.4}

Lemma 4.8. The map $\varphi: \mathrm{U}_{2}(C) \rightarrow \mathrm{SU}_{3}(C)$ given by $E \mapsto E \oplus \operatorname{det}(E)^{-1}$ is the normalization of its image, which is the singular locus of $\mathrm{SU}_{3}(C)$.

Proof. As a morphism to its image, $\varphi$ is quasi-finite and projective, and hence finite. Since $\varphi$ is also birational, it is the normalization map.

The stable locus of $\mathrm{SU}_{3}(C)$ is smooth, and the image of $\varphi$ is precisely the complement of the stable locus. Showing that the singular locus is all of the image reduces to showing that the generic point in the image of $\varphi$ has larger than expected tangent space; this follows by semicontinuity from characteristic 0 .

Lemma 4.9. The determinant map det: $\mathrm{U}_{2}(C) \rightarrow \mathrm{J}(C)$ induces an isomorphism of schemes $\operatorname{Alb}^{1}\left(\mathrm{U}_{2}(C)\right) \rightarrow \mathrm{J}(C)$.

Proof. Since det is a morphism to an abelian variety, it factors through the Albanese torsor, and it remains only to show that the induced morphism $\mathrm{Alb}^{1}\left(\mathrm{U}_{2}(C)\right) \rightarrow \mathrm{J}(C)$ is an isomorphism. The preimage of $0 \in \mathrm{J}(C)$ is a torsor over a subgroup scheme of $\operatorname{Alb}^{0}\left(\mathrm{U}_{2}(C)\right)$, so it remains to show that this torsor is a reduced point. The preimage of $0 \in \mathrm{J}(C)$ in $\operatorname{Alb}^{1}\left(\mathrm{U}_{2}(C)\right)$ agrees with the image in $\operatorname{Alb}^{1}\left(\mathrm{U}_{2}(C)\right)$ of $\mathrm{SU}_{2}(C)$. Since $\mathrm{SU}_{2}(C)$ is a smooth proper variety, the map $\mathrm{SU}_{2}(C) \rightarrow \mathrm{Alb}^{1}\left(\mathrm{U}_{2}(C)\right)$ factors through $\mathrm{Alb}^{1}\left(\mathrm{SU}_{2}(C)\right)$, and since $\mathrm{SU}_{2}(C) \cong \mathbb{P}^{3}$, the scheme $\mathrm{Alb}^{1}\left(\mathrm{SU}_{2}(C)\right)$ is a single point.

LEMma 4.10. If $X$ is a subvariety of $\mathbb{P}^{n}$ such that none of its irreducible components is contained in a hyperplane, then any element of $\mathrm{PGL}_{n+1}$ that fixes $X$ pointwise is trivial.

Proof. Pick $n+1$ points on $X$ which linearly span $\mathbb{P}^{n}$. For each subset of $n$ points, take the hyperplane spanned by them. By our assumptions, $X$ is not contained in the union of these $n+1$ hyperplanes. So take a point in $X$ outside of the union of these hyperplanes. This collection of $n+2$ points gives a projective coordinate frame. Indeed, if we lift the vectors to $k^{n+1}$, then there is a unique linear dependency up to scalar among these points and none of the coefficients can be 0 ; otherwise, we would have a contradiction with the construction of the last point. So any element of $\mathrm{PGL}_{n+1}$ that fixes $X$ pointwise also fixes these $n+2$ points and hence must be the identity.

Lemma 4.11. The automorphism group of $\mathrm{SU}_{3}(C)$ is finite.

Proof. We have homomorphisms

$$
\text { Aut }\left(\mathrm{SU}_{3}(C)\right) \rightarrow \operatorname{Aut}\left(\operatorname{Sing}\left(\mathrm{SU}_{3}(C)\right)\right) \rightarrow \operatorname{Aut}\left(\mathrm{U}_{2}(C)\right) \rightarrow \operatorname{Aut}\left(\operatorname{Alb}^{1}\left(\mathrm{U}_{2}(C)\right)\right) \cong \operatorname{Aut}(\mathrm{J}(C)),
$$

where the second map comes from Lemma 4.8 and the isomorphism comes from Lemma 4.9.

By Proposition 3.3, the group $\operatorname{Pic}\left(\mathrm{SU}_{3}(C)\right)$ is infinite cyclic, and only one of its generators has sections (for example, by Corollary 3.5), so any automorphism of $\mathrm{SU}_{3}(C)$ has to preserve $\mathcal{O}(1)$, and in particular the map $\theta$. In particular, Aut $\left(\mathrm{SU}_{3}(C)\right)$ is affine (being an extension with finite kernel of a linear group scheme). 


\section{VECTOR BUNDLES ON GENUS 2 CURVES AND TRIVECTORS}

So the image of $\operatorname{Aut}\left(\mathrm{SU}_{3}(C)\right)$ in $\operatorname{Aut}(\mathrm{J}(C))$ is finite, and it suffices to show that the kernel $K$ of this composition is finite. A point $E$ in $\mathrm{SU}_{3}(C)$ that splits as a sum of three line bundles $L_{1} \oplus L_{2} \oplus L_{3}$ is determined by the subscheme of $\mathrm{J}(C)$ which is the image of $\varphi^{-1}(E)$ (where $\varphi$ is the map in Lemma 4.8) in $\operatorname{Alb}^{1}\left(\mathrm{U}_{2}(C)\right.$ ) (geometrically, this consists of the points $\left\{L_{1} \otimes L_{2}, L_{2} \otimes\right.$ $\left.\left.L_{3}, L_{1} \otimes L_{3}\right\}\right)$. It therefore follows that $E$ is fixed by $K$, and hence $K$ pointwise fixes the locus $X$ of points in $\mathrm{SU}_{3}(C)$ representing vector bundles that are sums of three line bundles.

Note that $X$ is irreducible since it is the image of $\mathrm{J}(C) \times \mathrm{J}(C) \rightarrow \mathrm{SU}_{3}(C)$ under the map $\left(L_{1}, L_{2}\right) \mapsto L_{1} \oplus L_{2} \oplus\left(L_{1} \otimes L_{2}\right)^{-1}$. Furthermore, $\theta(X)$ is a reduced Heisenberg-invariant subscheme and so is not contained in any hyperplane of $\mathbb{P}^{8}$ : indeed, the given representation of the Heisenberg group is irreducible (even for non-ordinary curves of characteristic 3) by [Sek77, Appendix]. So by Lemma 4.10, any element of PGLg which fixes $\theta(X)$ is trivial, and hence $K$ is finite.

Proof of Proposition 4.4. First, we claim that the stabilizer in $\mathrm{SL}_{9}(k)$ is finite. For this, it suffices to show that the projective stabilizer of the line $\left[\gamma_{(C, P)}\right]$ in $\mathrm{PGL}_{9}(k)$ is finite. Note that any element in the projective stabilizer gives a linear change of coordinates of $\mathbb{P}^{8}$ which preserves the branch locus of $\theta$, and hence gives an automorphism of $\mathrm{SU}_{3}(C)$, so we can use Lemma 4.11 to finish the proof of our claim.

If $\mathrm{J}(C)[3] \cong \boldsymbol{\mu}_{3}^{2} \times(\mathbb{Z} / 3)^{2}$, then $\gamma_{(C, P)}$ is Heisenberg-invariant, so can be conjugated into the subspace $\mathfrak{h}$ spanned by the vectors in $[\mathrm{RS} 17,(2.4)]$, and it follows in particular that there exist such elements with finite stabilizer. Hence, by dimension considerations, if $U \subset \mathfrak{h}$ is the open subset on which the stabilizer is finite, then $\mathrm{SL}_{9} \cdot U$ is Zariski dense in $\bigwedge^{3}\left(V_{9}\right)$. It follows in particular that over any field, the generic point of $\mathfrak{h}$ is stable, and since the non-stable locus is everywhere of codimension at most 1 [RS17, Proposition 2.10], it follows that the non-stable locus in $\mathfrak{h}$ over $\mathbb{Z}$ is the Zariski closure of the characteristic 0 non-stable locus. The latter is a union of hyperplanes [RS17, Proposition 2.8], and thus the same holds over any field. Any element of such a hyperplane can clearly be lifted to characteristic 0 in such a way as to remain a non-stable element of $\mathfrak{h}$. In particular, the lift has infinite stabilizer, so the same holds for the original element.

It follows that $\gamma_{(C, P)}$ is stable for all but the codimension 2 or higher locus of non-ordinary curves of characteristic 3. Again, [RS17, Proposition 2.10] tells us that the non-stable locus is everywhere of codimension at most 1 and must therefore be empty in the moduli stack of pairs $(C, P)$ with $C$ smooth.

\section{Projective duality and Coble hypersurfaces}

Let $V_{9}$ be a 9-dimensional vector space. Fix a stable vector $\gamma \in \bigwedge^{3} V_{9}$ with respect to the natural action of $\mathrm{SL}\left(V_{9}\right)$. Let $\mathbb{P}\left(V_{9}^{*}\right)$ denote the space of lines in $V_{9}^{*}$. Apply the comultiplication map $\bigwedge^{3} V_{9} \rightarrow \bigwedge^{2} V_{9} \otimes V_{9}$ to $\gamma$, use the natural surjection $V_{9} \otimes \mathcal{O}_{\mathbb{P}\left(V_{9}^{*}\right)} \rightarrow \mathcal{O}_{\mathbb{P}\left(V_{9}^{*}\right)}(1)$, and interpret $\bigwedge^{2} V_{9}$ as the space of skew-symmetric matrices $V_{9}^{*} \rightarrow V_{9}$. This allows us to interpret $\gamma$ as a family of skew-symmetric matrices

$$
\Phi_{\gamma}: V_{9}^{*} \rightarrow V_{9} \otimes \mathcal{O}_{\mathbb{P}\left(V_{9}^{*}\right)}(1)
$$

over $\mathbb{P}\left(V_{9}^{*}\right)$. Let $X_{\gamma} \subset Y_{\gamma} \subset \mathbb{P}\left(V_{9}^{*}\right)$ be the loci

$$
X_{\gamma}=\left\{x \in \mathbb{P}\left(V_{9}^{*}\right) \mid \operatorname{rank}\left(\left.\Phi_{\gamma}\right|_{x}\right) \leqslant 4\right\}, \quad Y_{\gamma}=\left\{x \in \mathbb{P}\left(V_{9}^{*}\right) \mid \operatorname{rank}\left(\left.\Phi_{\gamma}\right|_{x}\right) \leqslant 6\right\} .
$$

Improving upon [GS15, GSW13], the following theorem is proven in [RS17, §3]. 


\section{E. M. RAINS AND S. V SAM}

Theorem 5.1. (a) The locus $Y_{\gamma}$ is a cubic hypersurface whose singular locus is $X_{\gamma}$.

(b) The locus $X_{\gamma}$ is smooth of dimension 2 , and the locus where rank $\Phi_{\gamma} \leqslant 2$ is empty.

(c) If $X_{\gamma}$ has a rational point, then it is isomorphic to the Jacobian of a smooth genus 2 curve $C_{\gamma}$. Furthermore, the line bundle giving the embedding $X_{\gamma} \subset \mathbb{P}\left(V_{9}^{*}\right)$ is a $(3,3)$-polarization.

In Definition 4.2, we constructed a sextic hypersurface $D_{\gamma} \subset \mathbb{P}\left(V_{9}\right)$.

Proposition 5.2. If $\gamma$ is stable, then $D_{\gamma}$ and $Y_{\gamma}$ are projectively dual hypersurfaces.

Proof. We may assume, without loss of generality, that $k$ is algebraically closed. We introduce an auxiliary variety. Let $F$ be the variety of flags of the form $V_{1} \subset V_{3} \subset V_{8} \subset V_{9}^{*}$ (the subscripts denote dimensions). Let $\mathcal{V}_{1} \subset \mathcal{V}_{3} \subset \mathcal{V}_{8} \subset \mathcal{V}_{9}=V_{9}^{*} \otimes \mathcal{O}_{F}$ denote the tautological flag of subbundles on $F$. Then $\bigwedge^{3} \mathcal{V}_{9}$ has a homogeneous subbundle $\xi$ which has a filtration by (homogeneous) subbundles whose associated graded bundle is

$$
\bigwedge^{3} \mathcal{V}_{3} \oplus\left(\mathcal{V}_{1} \otimes \mathcal{V}_{3} / \mathcal{V}_{1} \otimes \mathcal{V}_{9} / \mathcal{V}_{3}\right) \oplus\left(\bigwedge^{2}\left(\mathcal{V}_{3} / \mathcal{V}_{1}\right) \otimes \mathcal{V}_{8} / \mathcal{V}_{3}\right)
$$

Let $Z_{\gamma}$ be the subvariety of $F$ where the image of $\gamma$ under $\bigwedge^{3} \mathcal{V}_{9}^{*} \rightarrow \xi^{*}$ is 0 . Let $\pi_{1}: F \rightarrow \mathbb{P}\left(V_{9}^{*}\right)$ and $\pi_{2}: F \rightarrow \mathbb{P}\left(V_{9}\right)$ be the maps sending $V_{1} \subset V_{3} \subset V_{8}$ to $V_{1}$ and $V_{8}$, respectively.

Pick a point $x \in Z_{\gamma}$ and choose a basis $e_{1}, \ldots, e_{9}$ for $V_{9}$ such that $x$ is the standard coordinate flag $\left(V_{i}\right.$ is spanned by $\left.e_{1}^{*}, \ldots, e_{i}^{*}\right)$. Write $[i j k]$ in place of $e_{i} \wedge e_{j} \wedge e_{k}$. The conditions imposed by the composition $\bigwedge^{3} \mathcal{V}_{9}^{*} \rightarrow \xi^{*}$ being 0 are that the coefficients of the following monomials are 0 :

$$
[123], \quad[12 i],[13 i] \quad(4 \leqslant i \leqslant 9), \quad[23 j] \quad(4 \leqslant j \leqslant 8) .
$$

In particular, the $9 \times 9$ skew-symmetric matrix $\Phi_{\gamma}\left(\pi_{1}(x)\right)$ only has nonzero entries in the bottom right $6 \times 6$ corner, so has rank at most 6 , and so $\pi_{1}(x) \in Y_{\gamma}$. Conversely, given a point $y \in Y_{\gamma} \backslash X_{\gamma}$ ( $\operatorname{sorank} \Phi_{\gamma}(y)=6$ ), we may choose a basis such that this point is given by $e_{i}(y)=0$ for $i>1$ and the elements in its kernel satisfy $e_{i}=0$ for $i>3$. We may also arrange for the coefficients of [23j] to vanish for $j=4, \ldots, 8$. Then the standard coordinate flag in $F$ maps to $y$. So $\pi_{1}\left(Z_{\gamma}\right)=Y_{\gamma}$.

We claim that the tangent space to $y$ in $Y_{\gamma}$ is $V_{8} / V_{1}$. In our coordinates, $y=[1: 0: 0: 0$ : $0: 0: 0: 0: 0]$, so we work in the affine subspace given by $e_{1}=1$. The equation of the tangent space is $\sum_{i}\left(\partial f / \partial e_{i}\right)(0) e_{i}$, where $f$ is the Pfaffian of $\Phi_{\gamma}(y)$ after specializing $e_{1}=1$ (and deleting the first row and column). Let $M$ be this $8 \times 8$ skew-symmetric matrix (so $f=\operatorname{Pf}(M)$ ). Using the Laplace expansion for Pfaffians, we can compute $f$ by taking a signed sum of $M_{1, i} P_{i}$, where $2 \leqslant i \leqslant 8$ and $P_{i}$ is the Pfaffian of the result of deleting rows and columns 1 and $i$ from $M$. Note that $P_{i}(0)=0$ unless $i=2$ since the entries of $\Phi_{2}(y)$ are concentrated in the bottom right $6 \times 6$ matrix. Since $e_{1}$ does not appear in rows 1 and 2 (before specializing to $e_{1}=1$ ), this implies that the equation of the tangent space is $\sum_{i}\left(\partial M_{1,2} P_{2} / \partial e_{i}\right)(0) e_{i}$. From the constraints on $\gamma$ above, $M_{1,2}$ is the coefficient of [239] times $e_{9}$. In particular, the equation of the tangent space is a (nonzero, since $y$ is smooth) multiple of $e_{9}=0$, so the claim is proven.

In particular, $\pi_{1}$ is injective over the smooth locus (we have shown that $V_{3}$ and $V_{8}$ in any flag in $\pi_{1}^{-1}(y)$ are both determined by $y$ ). Since $Y_{\gamma}$ is normal (its singular locus $X_{\gamma}$ has codimension 5), this implies that $\pi_{1}$ is birational.

The image of $\gamma$ under the projection $\bigwedge^{3} \mathcal{V}_{9}^{*} \rightarrow \bigwedge^{3}\left(\mathcal{V}_{8}\right)_{x}^{*}$ has the property that $\gamma\left(v_{1}, v_{2}, v_{3}\right)=0$ whenever two of the vectors belong to $\left(\mathcal{V}_{3}\right)_{x}$. But $\mathcal{V}_{8}^{*}$ is the pullback along $\pi_{2}$ of the bundle $\mathcal{Q}$ in Definition 4.2, so this is the same trilinear form used there. In particular, the hyperdiscriminant of this form vanishes by Proposition 2.5, so $\pi_{2}(x) \in D_{\gamma}$. 


\section{VECTOR BUNDLES ON GENUS 2 CURVES AND TRIVECTORS}

Conversely, given a point $y \in D_{\gamma}$ whose associated 3-form has vanishing hyperdiscriminant, there is a 3 -dimensional space $V_{3}$ with the property that $\gamma\left(v_{1}, v_{2}, v_{3}\right)=0$ whenever two of the vectors belong to $V_{3}$ (Proposition 2.5). As above, we can pick a basis such that the standard coordinate flag lies in $Z_{\gamma}$ and maps to $y$ under $\pi_{2}$. So $\pi_{2}\left(Z_{\gamma}\right)=D_{\gamma}$.

In particular, we conclude that $D_{\gamma}$ is the closure of the set of tangent hyperplanes to smooth points of $Y_{\gamma}$, so the two hypersurfaces are projectively dual to one another.

Corollary 5.3. The hypersurfaces $Y_{\gamma}$ and $D_{\gamma}$ are irreducible.

Proof. If $Y_{\gamma}$ were reducible, then it would be singular in codimension 1, but we know that its singular locus is $X_{\gamma}$, which has codimension 5 in $Y_{\gamma}$. The projective dual of an irreducible variety is irreducible [GKZ08, $\S 1.1$, Proposition 1.3], so $D_{\gamma}$ is also irreducible.

TheOrem 5.4. Let $C$ be a smooth genus 2 curve. In characteristic different from 2 , the branch locus of $\theta: \mathrm{SU}_{3}(C) \rightarrow|3 \Theta|$ is a degree 6 hypersurface whose projective dual is a $\mathrm{J}(C)[3]$-invariant cubic hypersurface whose singular locus is a torsor for $\mathrm{J}(C)$.

Proof. We may assume as well that $k$ is algebraically closed. From Corollary 5.3, the branch locus is irreducible. Since the characteristic is different from 2, the irreducibility of $\mathrm{SU}_{3}(C)$ combined with this fact implies that the branch locus is a reduced hypersurface.

Pick any Weierstrass point $P \in C$. The construction in Section 4 gives a vector $\gamma_{(C, P)} \in$ $\bigwedge^{3} V_{9}$ with $V_{9}=\mathrm{H}^{0}\left(\mathrm{SU}_{3}(C) ; \Theta\right)^{*}$ such that $D_{\gamma_{(C, P)}}$ is the branch locus of $\theta$ (Proposition 4.3). Furthermore, $\gamma_{(C, P)}$ is stable by Proposition 4.4. By Proposition 5.2, the projective dual of $D_{\gamma_{(C, P)}}$ is a cubic hypersurface, and from Theorem 5.1, the singular locus of the cubic hypersurface is isomorphic to $\mathrm{J}(C)$.

Remark 5.5. In characteristics different from 3, it is easy to show using the techniques in [Bea03] that there is a unique $\mathrm{J}(C)[3]$-invariant cubic hypersurface whose singular locus is $\mathrm{J}(C)$. In particular, Theorem 5.4 generalizes the main result of [Ngu07, Ort05] to characteristics different from 2 and 3 .

Remark 5.6. We have seen how to construct the Coble cubic and its projective dual directly from $\gamma$. Using the equation (2.3), we can also build, directly from $\gamma$, a double cover of $\mathbb{P}\left(V_{9}\right)$ which is branched along $D_{\gamma}$. However, we also know that this double cover is $\operatorname{SU}_{3}(C)$, so we have an invariant-theoretic construction for it.

Finally, we make some comments about the case when $k$ is a field of characteristic 2 .

Lemma 5.7. Let $V$ be a vector space over a field of characteristic 2 , and let $k>1$ be an integer. Then there is a unique quadratic map $Q: \bigwedge^{k} V \rightarrow \bigwedge^{2 k} V$ which vanishes on pure tensors and satisfies $Q(v+w)=Q(v)+Q(w)+v \wedge w$.

Proof. The uniqueness is easy: a quadratic map is determined by the associated bilinear map and its values on a basis, and the standard basis consists entirely of pure tensors.

For the existence, define $Q$ by $Q\left(v_{1}+\cdots+v_{r}\right)=\sum_{1 \leqslant i<j \leqslant r} v_{i} \wedge v_{j}$ whenever the $v_{i}$ are pure tensors in the standard basis. It remains only to show that $Q$ vanishes on all pure tensors, not just those formed from coordinate vectors. This follows by induction on the number of coordinate vectors that appear in a pure tensor: expand one of the non-coordinate vectors in the wedge into coordinate vectors and observe that each term in the sum is annihilated by $Q$ and any two terms wedge to 0 . 


\section{E. M. RAINS AND S. V SAM}

Remark 5.8. If $k$ is a field of characteristic 2, the branch locus of the map $\theta: \mathrm{SU}_{3}(C) \rightarrow \mathbb{P}\left(V_{9}\right)$ is the square of a cubic equation due to Lemma 2.2. We will identify this cubic equation now.

Let $\mathfrak{e}_{8}$ be the split Lie algebra of type $\mathrm{E}_{8}$ over $k$. It has a $(\mathbb{Z} / 3)$-graded decomposition $\mathfrak{s l}\left(V_{9}\right) \oplus$ $\bigwedge^{3} V_{9} \oplus \bigwedge^{6} V_{9}$ (see [RS17, $\left.\S 2.2\right]$ ). Also, $\mathfrak{e}_{8}$ has a squaring map $x \mapsto x^{[2]}$ which induces a squaring map $\bigwedge^{3} V_{9} \rightarrow \bigwedge^{6} V_{9}$; this is the map $Q: \bigwedge^{3} V_{9} \rightarrow \bigwedge^{6} V_{9}$ defined in Lemma 5.7. Given a quotient $V_{9} \rightarrow V_{8}$, also use $Q$ to denote the squaring map $\bigwedge^{3} V_{8} \rightarrow \bigwedge^{6} V_{8}$. Then we have a commutative square

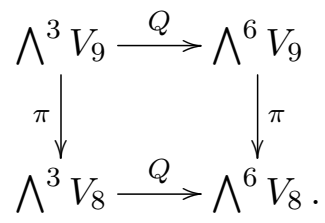

We claim that $f_{16}(\pi(\gamma))=\operatorname{Pf}(Q(\pi(\gamma)))^{2}$ (recall that $f_{16}$ is the hyperdiscriminant of $\bigwedge^{3} V_{8}$ ). Both $f_{16}$ and $(\mathrm{Pf} \circ Q)^{2}$ are degree 16 invariants; note that $\bigwedge^{3} V_{8}$ has a dense orbit with respect to $\mathrm{GL}\left(V_{8}\right)$, so by uniqueness, there is a constant $c$ such that $(\operatorname{Pf} \circ Q)^{2}=c f_{16}$. Since both are defined over $\mathbf{F}_{2}$, we have $c \in\{0,1\}$, so we just need to show that $\operatorname{Pf} \circ Q$ is not identically 0 . First note that $Q([123]+[456])=[123456]$, so $Q \neq 0$. It follows that if $\pi(\gamma)$ is an element in the dense orbit given by $(2.1)$, then $Q(\pi(\gamma)) \neq 0$. The stabilizer of this element in $\mathfrak{s l}\left(V_{8}\right)$ is $\mathfrak{s l}_{3}$, which acts irreducibly on $V_{8}$ (it is the adjoint representation), so we conclude that $Q(\pi(\gamma))$ has full rank, and so $\operatorname{Pf}(Q(\pi(\gamma))) \neq 0$. This shows that $c=1$ and proves the claim.

A point in $\mathbb{P}\left(V_{9}\right)$ represents a quotient $V_{9} \rightarrow V_{8}$, and it belongs to $D_{\gamma}$ if and only if $f_{16}(\pi(\gamma))=0$. From our claim above, this is equivalent to $\operatorname{Pf}(Q(\pi(\gamma))) \neq 0$.

So the cubic equation whose square is the branch locus of $\theta$ can be obtained by applying the Pfaffian construction (from the beginning of this section) to $\gamma^{[2]} \in \bigwedge^{3} V_{9}^{*} \cong \bigwedge^{6} V_{9}$.

\section{Appendix. Comparison}

Keep the notation from Section 3. In Section 4, we built a trivector $\gamma \in \bigwedge^{3} V_{9}$. Via comultiplication, we turn this into a map $V_{9}^{*} \rightarrow \bigwedge^{2} V_{9}$. Let $W_{\gamma} \subset \bigwedge^{2} V_{9}$ be its image. In [RS17, $\left.\S 4\right]$, we give an alternative description for $W_{\gamma}$. Our goal in this appendix is to prove Theorem A.1 so that we can show that this description agrees with the one here.

Let $V_{9}=\mathrm{H}^{0}\left(\mathrm{SU}_{3}(C) ; \mathcal{O}(1)\right)^{*}$ so that $\mathrm{J}^{1}(C) \subset \mathbb{P}\left(V_{9}^{*}\right)$ is embedded by a $(3,3)$-polarization, denoted by $\mathcal{O}(1)$. We identify $\mathrm{J}^{1}(C) \cong \mathrm{J}(C)$ via $\mathcal{L} \mapsto \mathcal{L}(-P)$. Define a codimension 1 subvariety of $\mathrm{J}(C) \times \mathrm{J}(C)$ by

$$
X=X_{C, P}=\left\{\left(\mathcal{L}_{1}, \mathcal{L}_{2}\right) \mid \operatorname{Hom}_{C}\left(\mathcal{L}_{1}, \mathcal{L}_{2}(P)\right) \neq 0\right\} .
$$

The line bundle $\mathcal{O}(1,1) \otimes \mathcal{O}(-X)$ has divisor class $3 \pi_{1}^{*} \Theta+3 \pi_{2}^{*} \Theta-\Theta_{\text {diag. }}$. This is the pullback of a principal polarization on $\mathrm{J}(C) \times \mathrm{J}(C)$ via the endomorphism

$$
\mathrm{J}(C) \times \mathrm{J}(C) \rightarrow \mathrm{J}(C) \times \mathrm{J}(C), \quad(a, b) \mapsto(2 a+b, a+2 b) .
$$

The kernel of this map is the diagonal copy of $\mathrm{J}(C)[3]$, which has degree 81. In particular, $\mathcal{O}(1,1) \otimes \mathcal{O}(-X)$ has a single cohomology group of dimension $9=\sqrt{81}$. We will see that it has nonzero global sections by exhibiting bilinear equations that vanish on $X$.

Theorem A.1. (a) $\operatorname{dim} W_{\gamma}=9$.

(b) The subspace $W_{\gamma} \subset \mathrm{H}^{0}(\mathrm{~J}(C) \times \mathrm{J}(C)$; $\mathcal{O}(1,1))$ vanishes on $X$. In particular,

$$
W_{\gamma}=\mathrm{H}^{0}(\mathrm{~J}(C) \times \mathrm{J}(C) ; \mathcal{O}(1,1) \otimes \mathcal{O}(-X)) .
$$




\section{VECTOR BUNDLES ON GENUS 2 CURVES AND TRIVECTORS}

If $\mathcal{L}$ is a line bundle of degree 0 on $C$, then the locus

$$
\Theta_{\mathcal{L} ; \mathrm{SU}_{3}(C)}=\left\{\mathcal{E} \in \mathrm{SU}_{3}(C) \mid \operatorname{Hom}_{C}(\mathcal{L}, \mathcal{E}(P)) \neq 0\right\}
$$

is a double cover of a hyperplane in $\mathbb{P}^{8}$. In particular, it is smooth away from the ramification locus, so that $\mathcal{L}$ determines a codimension 1 subspace of the tangent space at $\mathcal{E}$, and thus an element $\lambda_{\mathcal{L}}$ of the cotangent space (modulo scalars).

Generically, $\Theta_{\mathcal{E}}$ is a reduced divisor of $\mathrm{J}^{1}(C)$, and so $\operatorname{dim} \operatorname{Hom}(\mathcal{L}, \mathcal{E}(P))=1$. By Serre duality and the Riemann-Roch theorem, $\operatorname{dim} \operatorname{Hom}(\mathcal{E}, \mathcal{L}(P))=1$, and thus for generic $\mathcal{E}$, we have a unique (modulo scalars) composition

$$
\widetilde{\lambda}_{\mathcal{L}}: \mathcal{E} \rightarrow \mathcal{L}(P) \rightarrow \mathcal{E}(2 P) \cong \mathcal{E} \otimes \omega_{C} .
$$

Lemma A.3. Assume $\operatorname{dim} \operatorname{Hom}(\mathcal{E}, \mathcal{L}(P))=1$. The image of $\widetilde{\lambda}_{\mathcal{L}}$ under $\mathrm{H}^{0}\left(C ; \mathfrak{g l}(\mathcal{E}) \otimes \omega_{C}\right) \rightarrow$ $\mathrm{H}^{0}\left(C ; \mathfrak{p g l}(\mathcal{E}) \otimes \omega_{C}\right)$ is $\lambda_{\mathcal{L}}$ (up to a nonzero scalar).

Proof. A standard calculation with cocycles over the dual numbers (see [Muk95, Proposition 2.6]) shows that the tangent space of $\Theta_{\mathcal{L} ; \mathrm{SU}_{3}(C)}$ at $\mathcal{E}$ is the kernel of the cup product with any nonzero element in $\operatorname{Hom}(\mathcal{E}, \mathcal{L}(P))$ :

$$
\mathrm{H}^{1}(C ; \mathfrak{s l}(\mathcal{E})) \subset \operatorname{Ext}^{1}(\mathcal{E}, \mathcal{E}) \rightarrow \operatorname{Ext}^{1}(\mathcal{E}, \mathcal{L}(P)) .
$$

Twist the map $\mathcal{L}(P) \rightarrow \mathcal{E}(2 P)$ by $\omega_{C}^{-1}$; this gives a nonzero map $\operatorname{Hom}(\mathcal{E}, \mathcal{E}) \rightarrow \operatorname{Hom}(\mathcal{L}(-P), \mathcal{E})$, which is an isomorphism since both spaces are 1 -dimensional $(\operatorname{dim} \operatorname{Hom}(\mathcal{E}, \mathcal{E})=1$ since $\mathcal{E}$ is stable). By Serre duality, the dual map $\operatorname{Ext}^{1}(\mathcal{E}, \mathcal{L}(P)) \rightarrow \operatorname{Ext}^{1}\left(\mathcal{E}, \mathcal{E} \otimes \omega_{C}\right)$ is also an isomorphism. So being in the kernel of (A.4) is equivalent to being in the kernel of the cup product map with $\widetilde{\lambda}_{\mathcal{L}}$ :

$$
\mathrm{H}^{1}(C ; \mathfrak{s l}(\mathcal{E})) \subset \operatorname{Ext}^{1}(\mathcal{E}, \mathcal{E}) \rightarrow \operatorname{Ext}^{1}\left(\mathcal{E}, \mathcal{E} \otimes \omega_{C}\right) .
$$

In other words, $\widetilde{\lambda}_{\mathcal{L}}$ induces a linear functional on $\mathrm{H}^{1}(C ; \mathfrak{s l}(\mathcal{E}))$ whose kernel is the tangent space to $\Theta_{\mathcal{L} ; \mathrm{SU}_{3}(C)}$ and so agrees with $\lambda_{\mathcal{L}}$ up to nonzero scalar multiple.

LemmA A.5. Let $\mathcal{L}_{1}$ and $\mathcal{L}_{2}$ be line bundles such that $\operatorname{dim} \operatorname{Hom}\left(\mathcal{L}_{i}, \mathcal{E}(P)\right)=1$, so that the $\widetilde{\lambda}_{\mathcal{L}_{i}}(P)$ are defined.

(a) The map $\widetilde{\lambda}_{\mathcal{L}_{i}}(P)$ is a rank at most 1 endomorphism of the fiber $\mathcal{E}_{P}$.

(b) If $\operatorname{dim} \operatorname{Hom}\left(\mathcal{L}_{1}, \mathcal{L}_{2}(P)\right)=1$, then $\widetilde{\lambda}_{\mathcal{L}_{1}}(P)$ and $\widetilde{\lambda}_{\mathcal{L}_{2}}(P)$ commute.

Proof. Statement (a) is an immediate consequence of the fact that $\widetilde{\lambda}_{\mathcal{L}_{i}}$ factors through a line bundle.

Statement (b) is vacuous if $\mathcal{L}_{1} \cong \mathcal{L}_{2}$. Otherwise, it suffices to show that both compositions vanish. Consider the composition

$$
\mathcal{E} \rightarrow \mathcal{L}_{1}(P) \rightarrow \mathcal{E}(2 P) \rightarrow \mathcal{L}_{2}(3 P) \rightarrow \mathcal{E}(4 P) .
$$

Since $\mathcal{L}_{1} \neq \mathcal{L}_{2}$, the Riemann-Roch theorem gives $\operatorname{dim} \operatorname{Hom}\left(\mathcal{L}_{1}(P), \mathcal{L}_{2}(3 P)\right)=1$. By our assumption, it follows that the above composition factors through $\mathcal{L}_{2}(2 P) \rightarrow \mathcal{L}_{2}(3 P)$, which is 0 when restricted to $P$. The other composition is 0 by the same argument if we swap $\mathcal{L}_{1}$ and $\mathcal{L}_{2}$; we note that $\operatorname{dim} \operatorname{Hom}\left(\mathcal{L}_{2}, \mathcal{L}_{1}(P)\right)=1$ by Serre duality and the Riemann-Roch theorem.

Lemma A.6. For a generic pair $\left(\mathcal{L}_{1}, \mathcal{L}_{2}\right) \in \mathrm{J}(C) \times \mathrm{J}(C)$ with $\operatorname{dim} \operatorname{Hom}\left(\mathcal{L}_{1}, \mathcal{L}_{2}(P)\right)=1$, there exists a stable rank 3 bundle $\mathcal{E}$ with $\mathcal{E} \neq \iota^{*} \mathcal{E}^{*}$ such that $\operatorname{dim} \operatorname{Hom}\left(\mathcal{L}_{1}, \mathcal{E}(P)\right)=\operatorname{dim} \operatorname{Hom}\left(\mathcal{L}_{2}, \mathcal{E}(P)\right)=1$. 


\section{E. M. RAINS AND S. V SAM}

Proof. Let $X$ be the set of triples $\left(\mathcal{L}_{1}, \mathcal{L}_{2}, \mathcal{E}\right) \in \mathrm{J}(C) \times \mathrm{J}(C) \times \mathrm{SU}_{3}(C)$ where $\operatorname{Hom}\left(\mathcal{L}_{1}, \mathcal{L}_{2}(P)\right) \neq 0$, $\operatorname{Hom}\left(\mathcal{L}_{1}, \mathcal{E}(P)\right) \neq 0$, and $\operatorname{Hom}\left(\mathcal{L}_{2}, \mathcal{E}(P)\right) \neq 0$. Let $X_{0}$ be an irreducible component of $X$ whose image in $\mathrm{J}(C) \times \mathrm{J}(C)$ dominates the divisor $\left\{\left(\mathcal{L}_{1}, \mathcal{L}_{2}\right) \mid \operatorname{Hom}_{C}\left(\mathcal{L}_{1}, \mathcal{L}_{2}(P)\right) \neq 0\right\}$.

Now, let $\left(\mathcal{L}_{1}, \mathcal{E}\right)$ be a pair with $\operatorname{dim} \operatorname{Hom}\left(\mathcal{L}_{1}, \mathcal{E}(P)\right)=1$ and $\operatorname{dim} \operatorname{Hom}\left(\mathcal{E}, \iota^{*} \mathcal{E}^{*}\right)=0$ (such a pair exists, since each condition is separately a nonempty open condition on the locus $\left\{\left(\mathcal{L}_{1}, \mathcal{E}\right)\right.$ | $\left.\operatorname{Hom}\left(\mathcal{L}_{1}, \mathcal{E}(P)\right) \neq 0\right\}$ inside $\left.\mathrm{J}(C) \times \mathrm{SU}_{3}(C)\right)$. The loci $\left\{\mathcal{L}_{2} \mid \operatorname{Hom}\left(\mathcal{L}_{1}, \mathcal{L}_{2}(P)\right) \neq 0\right\}$ and $\left\{\mathcal{L}_{2} \mid\right.$ $\left.\operatorname{Hom}\left(\mathcal{L}_{2}, \mathcal{E}(P)\right) \neq 0\right\}$ are sections of $\Theta_{\mathcal{L}_{1}}$ and $3 \Theta$, respectively, and thus have intersection multiplicity $3 \Theta^{2}=6$. Moreover, there exists a section of $3 \Theta$ meeting the given section of $\Theta_{\mathcal{L}_{1}}$ at the point representing $\mathcal{L}_{1}$ with multiplicity 1 (take any hyperplane through $\mathcal{L}_{1}$ not containing the tangent vector), so this is true for the generic such section and can be imposed as a further nonempty open condition on $\mathcal{E}$. We thus see that any such pair $\left(\mathcal{L}_{1}, \mathcal{E}\right)$ can be extended to a triple $\left(\mathcal{L}_{1}, \mathcal{L}_{2}, \mathcal{E}\right)$ in $X_{0}$ (note that $\operatorname{Hom}\left(\mathcal{L}_{1}, \mathcal{L}_{2}(P)\right) \neq 0$ implies that $\left.\operatorname{dim} \operatorname{Hom}\left(\mathcal{L}_{1}, \mathcal{L}_{2}(P)\right)=1\right)$.

But then the locus in $X_{0}$ for which

$$
\operatorname{dim} \operatorname{Hom}\left(\mathcal{L}_{1}, \mathcal{L}_{2}(P)\right)=\operatorname{dim} \operatorname{Hom}\left(\mathcal{L}_{1}, \mathcal{E}(P)\right)=\operatorname{dim} \operatorname{Hom}\left(\mathcal{L}_{2}, \mathcal{E}(P)\right)=1
$$

and $\operatorname{dim} \operatorname{Hom}\left(\mathcal{E}, \iota^{*} \mathcal{E}^{*}\right)=0$ is an intersection of nonempty open sets and is thus (by irreducibility) nonempty.

Proof of Theorem A.1. (a) If not, then there is an 8-dimensional subspace $V_{8} \subset V_{9}$ such that $\gamma \in \bigwedge^{3} V_{8}$. In this case, $D_{\gamma}$ contains the hyperplane $\mathbb{P}\left(V_{8}\right)$. But this cannot happen: in odd characteristic, $D_{\gamma}$ is a reduced irreducible sextic by Corollary 5.3 and Theorem 5.4 , while in even characteristic, it is the square of a reduced irreducible cubic by Remark 5.8.

(b) A line bundle $\mathcal{L} \in \mathrm{J}(C)$ corresponds to a hyperplane in $V_{9}$. The preimage of this hyperplane is $\Theta_{\mathcal{L} ; \mathrm{SU}_{3}(C)}$, as defined in $(\mathrm{A} .2)$ (recall that we used $P$ to identify $\mathrm{J}(C)$ with $\mathrm{J}^{1}(C)$ ). So it suffices to show that for a generic choice of $\left(\mathcal{L}_{1}, \mathcal{L}_{2}\right)$, the linear map $\gamma\left(\lambda_{\mathcal{L}_{1}}, \lambda_{\mathcal{L}_{2}},-\right)$ is identically 0 . Furthermore, since $\gamma$ is alternating, it suffices to check this on the subspace $\Theta_{\mathcal{L}_{1} ; \mathrm{SU}_{3}(C)} \cap \Theta_{\mathcal{L}_{2} ; \mathrm{SU}_{3}(C)}$. By Lemma A.6, there is a stable bundle $\mathcal{E} \in \mathrm{SU}_{3}(C)$ with $\mathcal{E} \approx \iota^{*} \mathcal{E}^{*}$ such that

$$
\operatorname{dim} \operatorname{Hom}\left(\mathcal{L}_{1}, \mathcal{L}_{2}(P)\right)=\operatorname{dim} \operatorname{Hom}\left(\mathcal{L}_{1}, \mathcal{E}(P)\right)=\operatorname{dim} \operatorname{Hom}\left(\mathcal{L}_{2}, \mathcal{E}(P)\right)=1 .
$$

By Lemma A.5, the morphisms $\widetilde{\lambda}_{\mathcal{L}_{i}}: \mathcal{E} \rightarrow \mathcal{\varepsilon} \otimes \omega_{C}$ commute. So, for any element $\alpha \in \mathrm{H}^{0}(C ; \mathfrak{p g l}(\mathcal{E}) \otimes$ $\left.\omega_{C}\right)$, the section $\operatorname{Tr}\left(\left[\lambda_{\mathcal{L}_{1}}, \lambda_{\mathcal{L}_{2}}\right] \alpha\right)$ vanishes at $P$. So $\gamma\left(\lambda_{\mathcal{L}_{1}}, \lambda_{\mathcal{L}_{2}},-\right)$ vanishes for generic $\mathcal{E}$ in $\Theta_{\mathcal{L}_{1} ; \mathrm{SU}_{3}(C)} \cap \Theta_{\mathcal{L}_{2} ; \mathrm{SU}_{3}(C)}$, so we are done.

\section{REFERENCES}

Bar95 W. Barth, Quadratic equations for level-3 abelian surfaces, Abelian Varieties (Egloffstein, 1993) (de Gruyter, Berlin, 1995), 1-18.

Bea03 A. Beauville, The Coble hypersurfaces, C. R. Math. Acad. Sci. Paris 337 (2003), no. 3, 189-194; doi:10.1016/S1631-073X (03)00302-9.

Bho90 U. Bhosle, Pencils of quadrics and hyperelliptic curves in characteristic two, J. reine angew. Math. 407 (1990), 75-98; doi:10.1515/crl1.1990.407.75.

Cob17 A. B. Coble, Point sets and allied Cremona groups. III, Trans. Amer. Math. Soc. 18 (1917), no. 3, 331-372; doi:10.2307/1988959.

DN89 J.-M. Drezet and M.S. Narasimhan, Groupe de Picard des variétés de modules de fibrés semistables sur les courbes algébriques, Invent. Math. 97 (1989), no. 1, 53-94; doi:10.1007/ BF01850655. 


\section{VECTOR BUNDLES ON GENUS 2 CURVES AND TRIVECTORS}

DR76 U. V. Desale and S. Ramanan, Classification of vector bundles of rank 2 on hyperelliptic curves, Invent. Math. 38 (1976), no. 2, 161-185; doi:10.1007/BF01408570.

GKZ08 I. M. Gelfand, M. M. Kapranov, and A.V. Zelevinsky, Discriminants, resultants and multidimensional determinants, Mod. Birkhäuser Class. (Birkhäuser Boston, Inc., Boston, MA, 2008); doi:10.1007/978-0-8176-4771-1.

GS15 L. Gruson and S. V Sam, Alternating trilinear forms on a nine-dimensional space and degenerations of (3,3)-polarized Abelian surfaces, Proc. Lond. Math. Soc. 110 (2015), no. 3, 755-785; doi:10.1112/plms/pdu050.

GSW13 L. Gruson, S.V Sam, and J. Weyman, Moduli of abelian varieties, Vinberg $\theta$-groups, and free resolutions, Commutative Algebra (ed. I. Peeva) (Springer, New York, 2013), 419-469; doi:10.1007/978-1-4614-5292-8_13.

HL10 D. Huybrechts and M. Lehn, The geometry of moduli spaces of sheaves, second ed., Cambridge Math. Lib., Cambridge Uni. Press, Cambridge, 2010; doi:10.1017/CB09780511711985.

Hof12 N. Hoffmann, The Picard group of a coarse moduli space of vector bundles in positive characteristic, Cent. Eur. J. Math. 10 (2012), no. 4, 1306-1313; doi:10.2478/s11533-012-0064-0.

Lan04 A. Langer, Moduli spaces of sheaves in mixed characteristic, Duke Math. J. 124 (2004), no. 3, 571-586; doi:10.1215/S0012-7094-04-12434-0.

Las96 Y. Laszlo, Local structure of the moduli space of vector bundles over curves, Comment. Math. Helv. 71 (1996), no. 3, 373-401; doi:10.1007/BF02566426.

LR79 D. Luna and R.W. Richardson, A generalization of the Chevalley restriction theorem, Duke Math. J. 46 (1979), no. 3, 487-496; doi:10.1215/S0012-7094-79-04623-4.

Muk95 S. Mukai, Vector bundles and Brill-Noether theory, Current Topics in Complex Algebraic Geometry (Berkeley, CA, 1992/93), Math. Sci. Res. Inst. Publ., vol. 28 (Cambridge Univ. Press, Cambridge, 1995), 145-158.

New68 P.E. Newstead, Stable bundles of rank 2 and odd degree over a curve of genus 2, Topology 7 (1968), 205-215; doi:10.1016/0040-9383(68)90001-3.

Ngu07 Q. M. Nguyễn, Vector bundles, dualities and classical geometry on a curve of genus two, Internat. J. Math. 18 (2007), no. 5, 535-558; doi:10.1142/S0129167X07004230.

Ort05 A. Ortega, On the moduli space of rank 3 vector bundles on a genus 2 curve and the Coble cubic, J. Algebraic Geom. 14 (2005), no. 2, 327-356; doi:10.1090/S1056-3911-04-00387-X.

Pop13 M. Popa, Generalized theta linear series on moduli spaces of vector bundles on curves, in Handbook of Moduli, Vol. III, Adv. Lect. Math., vol. 26 (Int. Press, Somerville, MA, 2013), 219-255.

Ray82 M. Raynaud, Sections des fibrés vectoriels sur une courbe, Bull. Soc. Math. France 110 (1982), no. 1, 103-125; doi:10.24033/bsmf. 1955.

RS17 E. M. Rains and S. V Sam, Invariant theory of $\bigwedge^{3}(9)$ and genus 2 curves, 2017, arXiv:1702. 04840

Sek77 T. Sekiguchi, On projective normality of Abelian varieties. II, J. Math. Soc. Japan 29 (1977), no. 4, 709-727; doi:10.2969/jmsj/02940709.

SK77 M. Sato and T. Kimura, A classification of irreducible prehomogeneous vector spaces and their relative invariants, Nagoya Math. J. 65 (1977), 1-155; doi:10.1017/S0027763000017633.

Tev01 E. Tevelev, Projectively dual varieties, 2001, arXiv:math.AG/0112028.

VM08 T. E. Venkata Balaji and V. B. Mehta, Singularities of moduli spaces of vector bundles over curves in characteristic 0 and p, Michigan Math. J. 57 (2008), 37-42; doi:10.1307/mmj/1220879395.

Eric M. Rains rains@caltech.edu

Department of Mathematics, California Institute of Technology, Pasadena, CA, USA

Steven V Sam svs@math.wisc.edu

Department of Mathematics, University of Wisconsin, Madison, WI, USA 